\title{
STABILITY ANALYSIS OF THE CHAOTIC LORENZ SYSTEM WITH A STATE-FEEDBACK CONTROLLER
}

\author{
by \\ DANIEL C. JONES \\ (Under the Direction of Yan Wu)
}

\begin{abstract}
The Lorenz model is considered a benchmark system in chaotic dynamics in that it displays extraordinary sensitivity to initial conditions and the strange attractor phenomenon. Even though the system tends to amplify perturbations, it is indeed possible to convert a strange attractor to a non-chaotic one using various control schemes. In this work it is shown that the chaotic behavior of the Lorenz system can be suppressed through the use of a feedback loop driven by a quotient controller. The stability of the controlled Lorenz system is evaluated near its equilibrium points using Routh-Hurwitz testing, and the global stability of the controlled system is established using a geometric approach. It is shown that the controlled Lorenz system has only one globally stable equilibrium point for the set of parameter values under consideration.
\end{abstract}

INDEX WORDS: Chaos control, Global stability, Lorenz equations, Thermosyphon 2010 Mathematics Subject Classification: 34H10, 34D23, 37N10 


\title{
STABILITY ANALYSIS OF THE CHAOTIC LORENZ SYSTEM WITH A STATE-FEEDBACK CONTROLLER
}

\author{
by \\ DANIEL C. JONES
}

B.S. in Physics, Georgia Southern University, 2005

M.S. in Physics, University of Kentucky, 2007

A Thesis Submitted to the Graduate Faculty of Georgia Southern University in Partial Fulfillment of the Requirement for the Degree

MASTER OF SCIENCE

STATESBORO, GEORGIA

2009 
(C) 2009

Daniel C. Jones

All Rights Reserved 


\title{
STABILITY ANALYSIS OF THE CHAOTIC LORENZ SYSTEM WITH A STATE-FEEDBACK CONTROLLER
}

\author{
by \\ DANIEL C. JONES
}

\author{
Major Professor: Yan Wu \\ Committee: Xiezhang Li \\ Jimmy L. Solomon
}

Electronic Version Approved:

July 2009 


\section{ACKNOWLEDGMENTS}

I would like to thank Dr. Yan Wu for his persistent optimism, encouragement, and guidance. I greatly appreciate the many hours we spent collaborating on this project. I also wish to thank Bryan Nelsen for our enlightening conversations regarding thermosyphon physics, Chris Lowder for his assistance with computing resources, and Dr. Scott Kersey for help with formatting this document. Finally, I would like to thank my wife for believing in me and supporting me. 


\section{TABLE OF CONTENTS}

Page

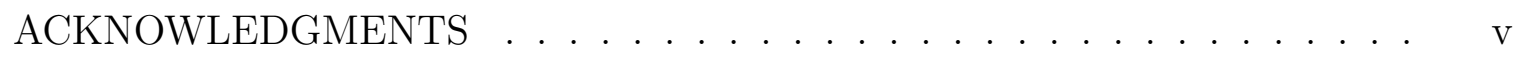

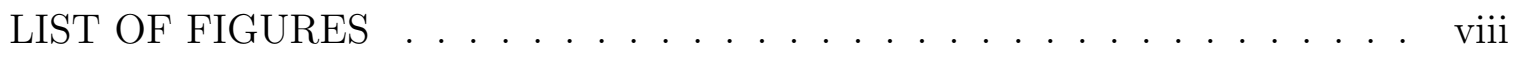

CHAPTER

1 Introduction ...................... 1

1.1 Single-loop Thermosyphon .............. . 1

1.2 Fluid Mechanics . . . . . . . . . . . . . . 3

1.3 Model of a Single-loop Thermosyphon . . . . . . . . . . 8

1.4 Classical, Deterministic Chaos ... . . . . . . 13

2 The Lorenz System . . . . . . . . . . . . . . . . . . . 20

2.1 The Lorenz Model . . . . . . . . . . . . . . . . . . . 20

2.2 Equilibrium Points . . . . . . . . . . . . . 23

2.3 Linear Approximation near Equilibrium Points . . . . . . 26

2.4 Stability near Equilibrium Points . . . . . . . . . . . 29

3 The Controlled Lorenz System . . . . . . . . . . . . . . . . . 38 
3.1 Quotient Controller . . . . . . . . . . . . . . . 38

$3.2 \quad$ Equilibrium Points . . . . . . . . . . . . . . . . 41

3.3 Local Stability of the Controlled Lorenz System _ . . . . 43

4 Global Stability . . . . . . . . . . . . . . . . . . 49

4.1 Introduction $\ldots \ldots \ldots \ldots \ldots \ldots$

4.2 Autonomous Convergence Theorems . . . . . . . . . 52

4.3 Global Stability Analysis . . . . . . . . . . . . . 55

$4.4 \quad$ Numerical Results . . . . . . . . . . . . . . . . . . 60

4.5 Conclusions and Future Work _ . . . . . . . . 61

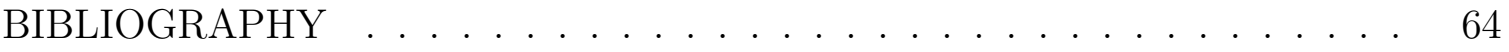

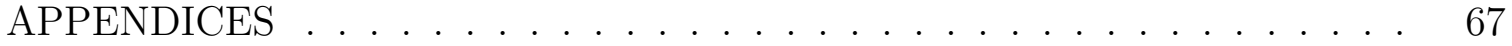

A Fourth-Order Runge-Kutta Method . . . . . . . . . . . 67

B MATLAB Code $\ldots \ldots \ldots \ldots \ldots$ 


\section{LIST OF FIGURES}

Figure

Page

1.1 Single-loop thermosyphon . . . . . . . . . . . . . . 2

1.2 Control volume . . . . . . . . . . . . . . . . . . . 4

1.3 Divergence of two nearby Lorenz trajectories . . . . . . . . . . . 17

1.4 Power spectrum of $x(t) \ldots \ldots \ldots \ldots$. . . . . . . . . . 18

1.5 System trajectory trapped on a strange attractor . . . . . . . . . . 19

2.1 Lorenz System with $\mathrm{R}=0$. . . . . . . . . . . . . . . 21

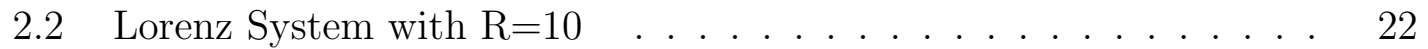

2.3 Lorenz System with $\mathrm{R}=28$. . . . . . . . . . . . . . . . . . 22

2.4 Behavior of the z-coordinate for $0<R<60 \ldots \ldots 24$

2.5 Behavior of the z-coordinate for $0<R<15 \ldots \ldots 25$

2.6 Stability of origin measured by $x \ldots \ldots 33$

2.7 Stability of equilibrium point $\left(x_{1}, y_{1}, z_{1}\right)$ measured by $z \quad \ldots . . .35$

2.8 Stability of equilibrium point $\left(x_{1}, y_{1}, z_{1}\right)$ measured by $x \ldots 36$ 


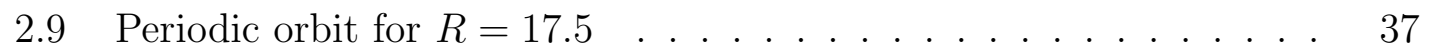

3.1 Controlled Lorenz system for $R=28$ and $k=5 \ldots . . . . . . .39$

3.2 Controlled Lorenz system for $R=28$ and $k=10$. . . . . . . . . 40

3.3 Controlled Lorenz system for $R=50$ and $k=15 \ldots . . . . . .40$

3.4 Behavior of $P_{2}(R, k) \ldots \ldots \ldots \ldots$

3.5 Regions of stability $\left(c_{2}>0\right)$ and instability $\left(c_{2}<0\right)$ for $x_{1} \ldots \ldots 4$

4.1 Lozinskii norm, $k=20, t_{f}=30 \ldots \ldots \ldots 2$

4.2 Lozinskii norm, $k=10, t_{f}=30 \ldots \ldots \ldots$ 


\section{CHAPTER 1 \\ INTRODUCTION}

In science and engineering, it is often necessary to consider the transfer of heat between bodies in thermal contact. Heat transfers are driven by temperature differences and are carried out by some combination of direct molecular collisions (conduction,) bulk motion of a fluid (convection,) and electromagnetic radiation. In this chapter, we discuss thermosyphon, a method of heat transfer which makes use of gravity-driven convection in a fluid-filled loop. After discussing some fundamental concepts from fluid dynamics, we construct a mathematical model of a thermosyphon device and note that the model reduces to the classical Lorenz system upon a nondimensionalization of variables.

\subsection{Single-loop Thermosyphon}

Thermosyphon is a method of heat transfer via convection of a fluid in a closed vertical loop. We may also call any device which makes use of this kind of convection a thermosyphon. One half of the loop is in thermal contact with a hot reservoir (a heat source) at a temperature $T_{h}$ and the other half is in thermal contact with a cold reservoir (a heat sink) at a temperature $T_{c}$. The average temperature between the two reservoirs is $T_{0}=\frac{1}{2}\left(T_{h}+T_{c}\right)$, and we use as a temperature difference parameter $\Delta T=\frac{1}{2}\left(T_{h}-T_{c}\right)$, such that $T_{c}=T_{0}-\Delta T$ and $T_{h}=T_{0}+\Delta T$. We take the shape of the loop to be a torus, with the radius from the center of the loop to the center of the torus tube written as $l$ and the radius of the torus tube written as $r$, where $r \ll l$. Under this assumption, we can neglect variations in fluid velocity, pressure, density, and temperature along the tube radius $r$ by taking cross-sectional 
averages of the quantities of interest at each angular position $\phi$. A simple diagram of a thermosyphon is shown in Figure 1.1, where the inscribed angle is $\phi$ and $x, y$, and $z$ are state variables proportional to the fluid flow velocity, horizontal temperature difference, and vertical temperature difference, respectively.

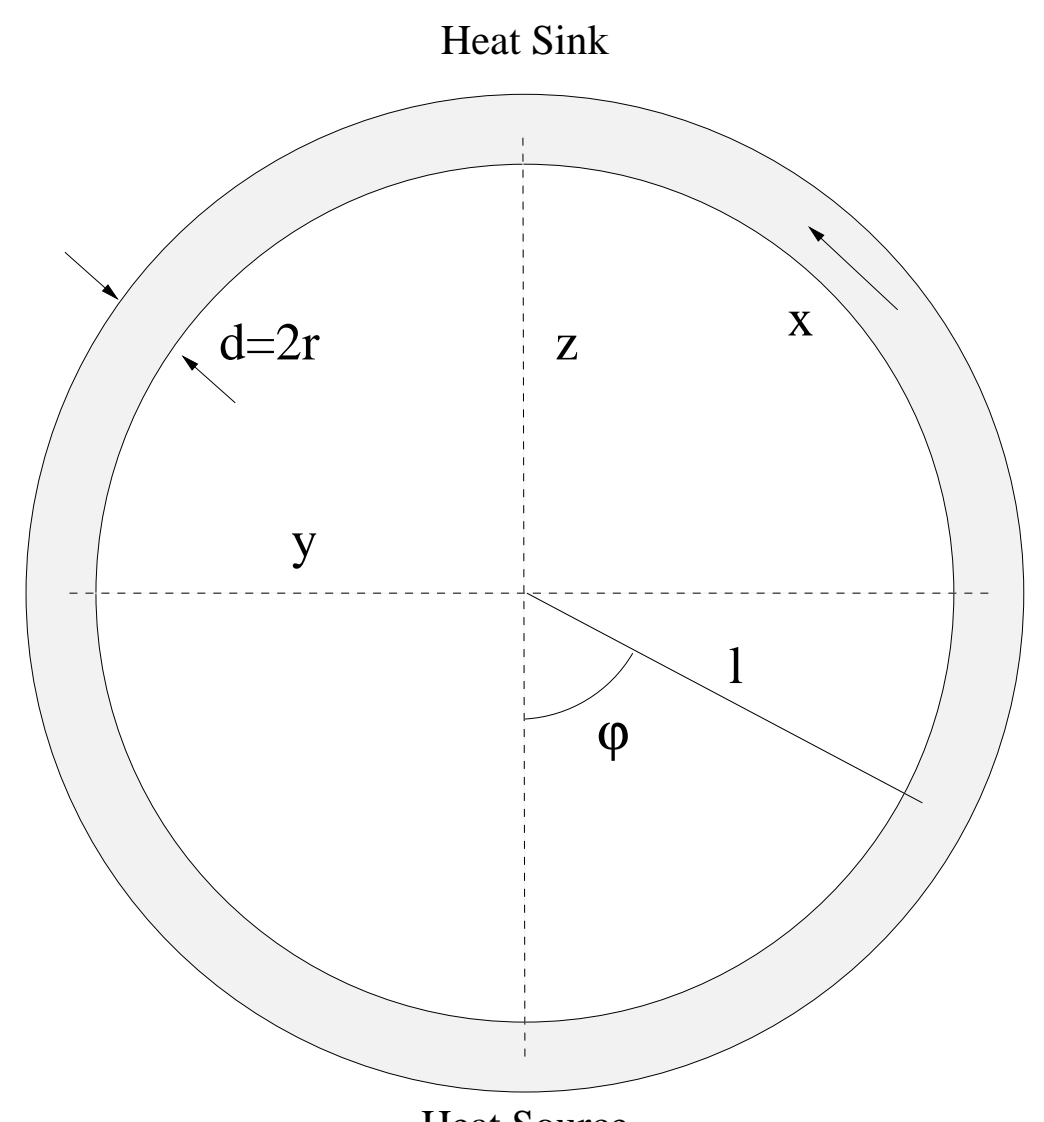

Heat Source

Figure 1.1: Single-loop thermosyphon

For small values of $\Delta T$, heat transfer is dominated by conduction, which is the diffusion of thermal energy via collisions between the molecules that make up the fluid. At a critical value $\Delta T_{\text {crit }}$, the state of rest becomes unstable and convection begins in either the clockwise or counterclockwise direction. In this regime, we may say that momentum diffusivity dominates thermal diffusivity, or that conduction alone is 
insufficient to transport thermal energy from the heat source to the heat sink through the fluid at the rate required for equilibrium. For even higher values of $\Delta T$, the thermosyphon undergoes another qualitative change in behavior (called a bifurcation) as the fluid flow becomes chaotic - the speed changes erratically and the direction of fluid flow reverses at unpredictable times. While physically interesting, this is usually undesirable from an engineering perspective as chaotic fluid flow in a thermosyphon results in an unpredictable flow of thermal energy as well as vibrations and additional fatigue on the device. To better understand the properties of the thermosyphon, we need a quantitative model built on the foundations of fluid mechanics.

\section{$1.2 \quad$ Fluid Mechanics}

We start with a broad description of fluid mechanics partly adapted from [1]. Fluid mechanics is the theory of flow in those liquids (hydrodynamics) and gases (aerodynamics) that can be meaningfully described by the continuum approximation. Under the continuum approximation, one assumes that even the smallest volume considered will contain so many molecules that we can safely ignore the fact that the fluid is made up of discrete particles and instead use average properties over these small volumes. In this way we can assign to any point $Q$ in the fluid a mean velocity $\mathbf{v}$ of the particles in a small volume element around $Q$. The resulting velocity distribution in space and time is called the velocity field $\mathbf{v}=\mathbf{v}(\mathbf{r}, t)$, where $\mathbf{r}$ is the position vector. Analogously, we can also introduce the pressure field $P=P(\mathbf{r}, t)$, the temperature field $T=T(\mathbf{r}, t)$ and the density field $\rho=\rho(\mathbf{r}, t)$. Note that this description only holds when the system is in local thermodynamic equilibrium, since that is when pressure and temperature may be meaningfully defined [1]. 
The equations that govern the temporal and spatial evolution of these fields can be formulated in terms of three fundamental conservation laws from classical mechanics and thermodynamics, namely, we need the laws of

1. conservation of mass,

2. conservation of linear momentum, and

3. conservation of energy.

All three of these conservation laws can be written in terms of a continuity equation derived from the Reynolds transport theorem. A more thorough treatment of fluid mechanics may be found in [17], [24], and [25], around which the following treatment was built. Here we only provide a rough outline of the results in order to motivate our model of the thermosyphon.

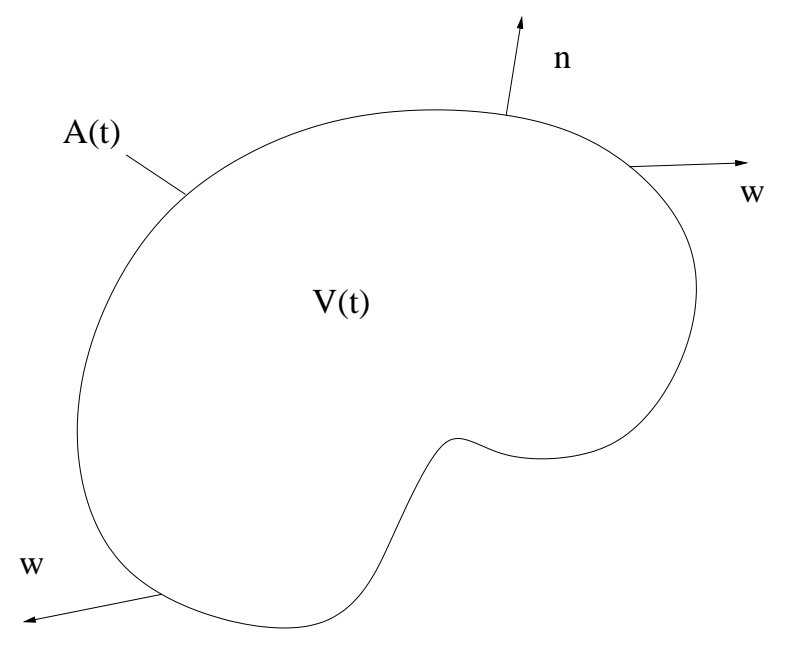

Figure 1.2: Control volume

Consider a control volume $V(t)$ with surface area $A(t)$ such as that shown in Figure 1.2. Points on the surface of the volume move with a velocity $\mathbf{w}$, and $\mathbf{n}$ is a 
vector that is everywhere normal to the surface. The time derivative of the volume integral of some scalar function $S$ is then given by

$$
\frac{d}{d t} \int_{V(t)} S d V=\int_{V(t)} \frac{\partial S}{\partial t} d V+\oint_{A(t)} S \mathbf{w} \cdot \mathbf{n} d A .
$$

In the special case where the volume is fixed in space, $\mathbf{w}=\mathbf{0}$ and

$$
\frac{d}{d t} \int_{V} S d V=\int_{V} \frac{\partial S}{\partial t} d V
$$

where the time derivative $d / d t$ is taken with respect to a fixed coordinate system. If we let the control volume move with the velocity of the fluid $\mathbf{v}$, we get the Reynolds transport theorem,

$$
\frac{d}{d t} \int_{V(t)} S d V=\int_{V(t)} \frac{\partial S}{\partial t} d V+\oint_{A(t)} S \mathbf{v} \cdot \mathbf{n} d A
$$

or if the quantity of interest is a vector $\mathbf{v}$,

$$
\frac{d}{d t} \int_{V(t)} \mathbf{v} d V=\int_{V(t)} \frac{\partial \mathbf{v}}{\partial t} d V+\oint_{A(t)} \mathbf{v}(\mathbf{v} \cdot \mathbf{n}) d A
$$

We can apply the Reynolds transport theorem to the mass of the system to get a continuity equation. The mass $M$ contained in a control volume $V(t)$ is given by

$$
M=\int_{V(t)} \rho d V
$$

The law of conservation of mass states that that $M$ must be a constant for a closed system,

$$
\frac{d M}{d t}=\frac{d}{d t} \int_{V(t)} \rho d V=0 .
$$

Equation (1.1) then becomes

$$
\frac{d}{d t} \int_{V(t)} \rho d V=\int_{V(t)} \frac{\partial \rho}{\partial t} d V+\oint_{A(t)} \rho \mathbf{v} \cdot \mathbf{n} d A=0
$$


The divergence theorem from vector calculus relates the volume integral of the divergence of a vector field $\mathbf{B}$ to the flux through a closed surface $A$ that bounds the volume $V$ by

$$
\int_{V} \nabla \cdot \mathbf{B} d V=\oint_{A} \mathbf{B} \cdot \mathbf{n} d A
$$

Applying this to (1.2), we get

$$
\frac{d}{d t} \int_{V(t)} \rho d V=\int_{V(t)}\left[\frac{\partial \rho}{\partial t}+\nabla \cdot(\rho \mathbf{v})\right] d V=0 .
$$

Since the volume integral in the middle expression must be zero for any arbitrary control volume $V(t)$, the term in brackets must be zero. This produces the continuity equation for mass per unit volume,

$$
\frac{\partial \rho}{\partial t}+\nabla \cdot(\rho \mathbf{v})=0
$$

which applies to every point in space. Note that

$$
\nabla \cdot(\rho \mathbf{v})=\mathbf{v} \cdot \nabla \rho+\rho \nabla \cdot \mathbf{v}
$$

so that for an incompressible flow, where $\nabla \cdot \mathbf{v}=0$, (1.3) becomes

$$
\frac{D \rho}{D t}=\frac{\partial \rho}{\partial t}+\mathbf{v} \cdot \nabla \rho=0
$$

The total time derivative operator

$$
\frac{D}{D t}=\frac{\partial}{\partial t}+\mathbf{v} \cdot \nabla
$$

is known as the material derivative with respect to time. The first term is called the local derivative and the second term is associated with the change of a quantity due to movement of the particle [25]. The symbol $d / d t$ is also used for the material derivative. 
Using a similar argument we can construct the continuity equation for linear momentum per unit volume,

$$
\frac{D(\rho \mathbf{v})}{D t}=\frac{\partial(\rho \mathbf{v})}{\partial t}+\mathbf{v} \cdot \nabla(\rho \mathbf{v})=\mathbf{F}
$$

where $\mathbf{F}$ is the force per unit volume acting on the system. This is an application of the fundamental law of mechanics, namely that the net force acting on a fluid mass enclosed in an arbitrary volume is equal to the time rate of change of the linear momentum of the fluid in that volume. The force per unit volume $\mathbf{F}$ may be divided into an external force $\mathbf{F}_{e}$ that acts on each point in the body, a surface force that takes the form of a pressure gradient $-\nabla P$, and a term $\eta \nabla^{2} \mathbf{v}$ that takes internal fluid friction into account [25]. The result is a variant of the Navier-Stokes equation,

$$
\frac{D(\rho \mathbf{v})}{D t}=-\nabla P+\mathbf{F}_{\mathbf{e}}+\eta \nabla^{2} \mathbf{v}
$$

which governs momentum transport in fluids.

An application of Reynolds theorem to internal energy produces a continuity equation for internal energy per unit volume $U$ in which we neglect phase changes such that $U=\rho c_{p} T$. If $H$ is the rate of thermal energy exchange between the thermosyphon and the environment, the continuity equation is

$$
\frac{D\left(\rho c_{p} T\right)}{D t}=\frac{\partial\left(\rho c_{p} T\right)}{\partial t}+\mathbf{v} \cdot \nabla\left(\rho c_{p} T\right)=H
$$

where $T$ is the temperature and $c_{p}$ is the specific heat capacity. In our case the fluid in the loop is held at constant volume, so the fluid does no work and changes in total internal energy are completely determined by heat flow $H$. 


\subsection{Model of a Single-loop Thermosyphon}

Now that we have established continuity equations for both momentum and internal energy, we can construct a mathematical model of a single-loop thermosyphon device. The continuity equation for linear momentum per unit volume, called the NavierStokes equation, is

$$
\rho\left[(\mathbf{v} \cdot \nabla) \mathbf{v}+\frac{\partial \mathbf{v}}{\partial t}\right]=-\nabla P+\mathbf{F}_{\mathbf{e}}+\eta \nabla^{2} \mathbf{v}
$$

From this point on, we take $\eta$ to be negligibly small, in which case (1.6) reduces to Euler's equation.

Since we are dealing with a toroidal fluid loop with $r \ll l$, we only care about the angular component of the velocity $v_{\phi}$. Let $u$ be the velocity component $v_{\phi}$ averaged over a cross-section through the torus tube. The angular component of the Euler equation is then

$$
\rho\left[u \frac{1}{l} \frac{\partial u}{\partial \phi}+\frac{\partial u}{\partial t}\right]=-\frac{1}{l} \frac{\partial P}{\partial \phi}+F_{\phi} .
$$

We can take the flow to be incompressible, or $\partial u / \partial \phi=0$. As a result, in this model the fluid will move as a whole, since every cross section of the fluid will have the same average velocity $u$ at each instant in time. Note that this is not the same as assuming that the fluid is completely incompressible, which would require us to neglect any variations in density and eliminate buoyancy-driven convection from our model. This is obviously undesirable, as convection is exactly what we want to study.

Instead we will use the Boussinesq approximation, which is frequently used in the study of convection. We assume that the fluid is incompressible except where thermal expansion gives rise to buoyancy. To say it another way, differences in inertia between different parts of the fluid are negligible but the weight per unit volume may vary. The 
only place we allow density variations is in the gravitational force, which preserves the buoyant mechanism for convection but ignores the equivalence of gravitational and inertial mass. Note that sound waves are by definition not allowed under the Boussinesq approximation.

The angular component of the gravitational force per unit volume acting on the fluid is $\rho g \sin (\phi)$, and we take the frictional force $f_{w}$ acting on the fluid to be proportional to the velocity $u$. As such, Euler's equation becomes

$$
\rho_{0} \frac{\partial u}{\partial t}=-\frac{1}{l} \frac{\partial P}{\partial \phi}-\rho(T) g \sin (\phi)-\frac{1}{2} \rho_{0} f_{w 0} u
$$

where $\rho_{0}$ is the fluid mass density at the reference temperature $T_{0}$ and $f_{w 0}$ is the coefficient of friction. Note that the dynamics of the fluid are coupled to its temperature profile through both the density and the pressure, so for a complete description we need a thermodynamical equation of state $f(\rho, P, T)=0$ and an equation governing the transport of thermal energy.

We must make some assumptions about the relationships between density, pressure, and temperature. First, we assume that density and pressure are linearly proportional at constant temperature, such that an increase in density corresponds to an increase in pressure. We also only allow a linear dependence of density on temperature. Expanding $\rho(T)$ in a Taylor series about the reference temperature $T_{0}$ and keeping only the linear term, we have

$$
\rho(T)=\rho_{0}\left[1-\alpha_{0}\left(T-T_{0}\right)\right]
$$

where

$$
\alpha_{0}=-\left.\frac{1}{\rho_{0}} \frac{\partial \rho}{\partial T}\right|_{\rho_{0}}
$$

is the coefficient of thermal expansion for the fluid in the loop. Note that since the 
volume of the fluid is fixed by the size of the loop, an increase in density of one part of the fluid must be accompanied by a decrease in density in some other part.

We assume that the work done by friction makes a negligible contribution to the fluid's internal energy. As such, the system's internal energy only changes by heat flow between the fluid and the environment. The rate of heat transfer $H=H(\phi, t)$ is proportional to the temperature difference between the fluid and the walls,

$$
H=h_{w}\left[T_{w}(\phi)-T(\phi)\right]
$$

where $h_{w}$ is the coefficient of conductive heat transfer at the loop wall, $T=T(\phi, t)$ is the fluid temperature, and $T_{w}(\phi)$ is the wall temperature. Using this expression in (1.5), we get

$$
\rho_{0} c_{p}\left(\frac{\partial T}{\partial t}+u \frac{1}{l} \frac{\partial T}{\partial \phi}\right)=h_{w}\left[T_{w}(\phi)-T(\phi)\right],
$$

The temperature at the wall is fixed by the presence of a heat source and a heat sink,

$$
T_{w}(\phi)=T_{0}+\Delta T \cos (\phi)
$$

such that the wall temperature at $\phi=0$ is $T_{h}=T_{0}+\Delta T$ and the temperature at $\phi=\pi$ is $T_{c}=T_{0}-\Delta T$.

We can expand the fluid temperature in a Fourier series of the form

$$
T(\phi, t)=T_{0}+\sum_{n=1}^{\infty}\left[S_{n}(t) \sin (n \phi)+C_{n}(t) \cos (n \phi)\right] .
$$

We keep only the leading terms, leaving

$$
T(\phi, t)=T_{0}+S_{1} \sin (\phi)+C_{1} \cos (\phi)
$$

This truncation is sometimes called the Galerkin procedure. While this method makes the model simpler to work with, it restricts the range in parameter space where the 
Lorenz model accurately reflects the behavior of real fluid flow. If the real fluid motion takes on a more complex structure, as it will if the temperature difference between the top and the bottom of the loop gets too large, then the Lorenz equations no longer provide a useful model of the dynamics [8]. As such, in this work we will restrict our attention to parameter values where higher-order spatial modes in the temperature distribution are unimportant.

To summarize up to this point, the dynamics of the thermosyphon are described by the system

$$
\begin{array}{r}
\rho_{0} \frac{\partial u}{\partial t}=-\frac{1}{l} \frac{\partial P}{\partial \phi}-\rho_{0}\left[1-\alpha_{0}\left(T-T_{0}\right)\right] g \sin (\phi)-\frac{1}{2} \rho_{0} f_{w 0} u \\
\rho_{0} c_{p}\left(\frac{\partial T}{\partial t}+u \frac{1}{l} \frac{\partial T}{\partial \phi}\right)=h_{w}\left[T_{0}+\Delta T \cos (\phi)-T(\phi)\right]
\end{array}
$$

where $T$ can be expanded in a truncated Fourier series. While this pair of equations is not amenable to direct solution, it was shown in [5] that the equations which govern the thermosyphon can be reduced to those of the Lorenz model under a transformation to the dimensionless variables

$$
\left\{\begin{aligned}
t^{\prime} & =\frac{h_{w}}{\rho_{0} c_{p}} t \\
x & =\frac{\rho_{0} c_{p}}{h_{w} l} u \\
y & =\kappa S_{1} \\
z & =\kappa\left(\Delta T-C_{1}\right),
\end{aligned}\right.
$$

where

$$
\kappa=\frac{\rho_{0} c_{p} \alpha_{0} g}{h_{w} l f_{w 0}} .
$$

Intuitively, $x$ is proportional to the fluid circulation speed, $y$ is proportional to the horizontal temperature difference (between $\phi=\frac{\pi}{2}$ and $\phi=\frac{3 \pi}{2}$ ) and $z$ to the vertical temperature difference (between $\phi=0$ and $\phi=\pi$ ). The details of implementing this 
transformation can be found in reference [23]. Upon making the above substitution, we get a set of three coupled first-order nonlinear differential equations

$$
\left\{\begin{array}{l}
\dot{x}=p(y-x) \\
\dot{y}=R x-y-x z \\
\dot{z}=x y-b z
\end{array}\right.
$$

where $\dot{x}=d x / d t^{\prime}, \dot{y}=d y / d t^{\prime}, \dot{z}=d z / d t^{\prime}$ and

$$
p=\frac{\rho_{0} c_{p}}{2 h_{w}} f_{w 0}
$$

The parameter $p$ is analogous to the Prandtl number from fluid dynamics, a measure of the relative importance of viscosity to thermal diffusivity of a fluid. A great deal of work has been done in which $p=10$, including that of Lorenz himself, so for the remainder of this work we take $p=10$. The number $R$, which we will treat as a tunable parameter, is given by

$$
R=\kappa \Delta T=\frac{\rho_{0} c_{p} \alpha_{0} g}{h_{w} l f_{w 0}} \Delta T
$$

where $R$ is actually the ratio of the Rayleigh number $R_{a}$ to its critical value $R_{c}$, such that $R=R_{a} / R_{c}$. When $R<1$ conduction is the dominant mechanism of heat transfer. When $R$ crosses 1 , convection begins. As we will see, as $R$ increases further the system transitions into the chaotic regime and the fluid flow becomes erratic. The parameter $b$ is analogous to the Biot number, which in fluid dynamics relates the resistance to heat transfer at the surface of a body to that inside the system. In this work, we assume that heat flow encounters the same resistance at the loop wall as it does in the fluid, such that $b=1$.

The system (1.16) is the classical Lorenz model, one of the first systems known to display extraordinary sensitivity to small modifications. It originally arose in Lorenz's 
study of forced dissipative hydrodynamic flow - more specifically in atmospheric convection cells. Upon observing the model's strong dependence on initial conditions, Lorenz concluded that long-term weather forecasting is impossible, since in even the simplest model of atmospheric behavior, "slightly differing initial states can evolve into considerably different states" [12].

To get more physical insight into the system, it may be helpful to write down the temperature and density in terms of our new variables. We can write a dimensionless temperature parameter $T_{s}$ of the fluid in the loop, with respect to the reference temperature $T_{0}$, as

$$
T_{s}(\phi, t)=\frac{T(\phi, t)-T_{0}}{\kappa}=y(t) \sin (\phi)+[R-z(t)] \cos (\phi)
$$

and the scaled dimensionless density parameter $\rho_{s}$ as

$$
\rho_{s}(\phi, t)=\frac{\rho(\phi, t)-\rho_{0}}{\alpha_{0} \rho_{0} \kappa}=-y(t) \sin (\phi)-[R-z(t)] \cos (\phi),
$$

so clearly $\rho_{s}(\phi, t)=-T_{s}(\phi, t)$, as expected. An increase in temperature corresponds to a decrease in density, and visa versa. Keep in mind that the temperature and density functions depend implicitly on the fluid flow velocity $x$ through the Lorenz equations (1.16).

\subsection{Classical, Deterministic Chaos}

In this section we give a description of classical chaos adapted from [2]. The Lorenz model is part of a larger class of dissipative systems, since it may lose energy to the external world over time. Such systems are deterministic, since they can be described by a continuous flow (in a mathematical sense, not that of fluid flow),

$$
\frac{d \mathbf{x}(t)}{d t}=F(\mathbf{x}(t))
$$


or by a discrete time mapping

$$
\mathbf{x}_{k+1}=f\left(\mathbf{x}_{k}\right)
$$

where $\mathbf{x}$ is a vector in $\mathbb{R}^{m}$. The mathematical space whose axes are the components of $\mathbf{x}$ is a phase space, in which each point in the space corresponds to a unique state of the system. A phase trajectory is a path through phase space which represents the evolution of the system, and a set of phase trajectories constitutes a phase portrait.

We are interested in the long-term behavior of dissipative systems. With some dissipative systems, it is possible to show that the trajectories stay in a bounded volume of phase space. That is, no system trajectories diverge to infinity. If this condition holds, then in this finite volume the trajectories will converge onto a geometrical object called an attractor, which is a compact set in phase space which is invariant under the flow or mapping [2]. For example, the trajectories of the Lorenz system do stay in a bounded volume of phase space. The divergence of the velocity field is always negative,

$$
\nabla \cdot \mathbf{v}=\frac{\partial \dot{x}}{\partial x}+\frac{\partial \dot{y}}{\partial y}+\frac{\partial \dot{z}}{\partial z}=-p-b-1<0
$$

thus the trajectories of the Lorenz system converge onto some kind of attractor. The nature of the attractor will depend on the values of the system parameters, though we can say in general that the attractor for a dissipative system like the Lorenz system has zero volume, since any volumes in space phase contract under the flow. The set of initial conditions giving rise to trajectories that converge onto this attractor is called the basin of attraction, which may have finite or infinite volume.

The simplest kind of attractor is the point attractor, which corresponds to a state which is independent of time. If the trajectory is periodic, with a single fundamental frequency and perhaps a few harmonics, the attractor is called a limit cycle. The 
solution can be written as a Fourier series, so it is possible to predict the behavior of the system for arbitrarily long times. If the attractor is a torus in $T^{r}$, with $r \geq 2$, the solution is called quasiperiodic with $r$ independent fundamental frequencies. While the solution cannot be written as a simple Fourier series, it is still possible to predict the solution for arbitrarily long times. Note that a dissipative system such as the Lorenz system cannot have a torus as an attractor, since a torus has a nonzero volume in phase space.

In the Lorenz system we find the earliest known example of a strange attractor, which is an attractor that displays an unpredictable, apparently non-repeating pattern. Trajectories on a strange attractor always diverge, even if they start very close to one another. It is this sensitive dependence on initial conditions that gives us a pseudo-definition for chaos. That is, we can call a dynamical regime chaotic when it displays extraordinary sensitivity to initial conditions and trajectories which appear unpredictable and noisy, such that its power spectrum contains, along with possible peaks, a broad band or continuous part [2].

Shown in Figure 1.3 is an example of two Lorenz trajectories which start quite close to one another but diverge after a sufficient amount of time has passed, illustrating the system's sensitivity to initial conditions. We see in Figure 1.4 that the power spectrum of $x(t)$ does indeed contain a broad band, though there are several sharp peaks corresponding to unstable periodic orbits present in phase space. Shown in Figure 1.5 is a single Lorenz system trajectory that quickly becomes trapped on the strange attractor.

Note that the appearance of unpredictability does not imply that the system is truly random. A chaotic system may exhibit somewhat periodic motion for a short 
time before switching to another apparently periodic behavior with a different period and envelope. It is this rapid switching between different nearly periodic behaviors that gives rise to the appearance of unpredictability, though the evolution remains fully deterministic in nature with no truly random elements. We can understand deterministic chaos as the superposition of an extremely large number of periodic motions, such that the onset of chaos must be accompanied by an exponential increase in the number of periodic motions available to a system [2]. Since a chaotic system has a very large number of periodic motions available to it in a bounded region of phase space, only a small perturbation is required to shift the system from one periodic orbit to another - this is the origin of a chaotic system's extraordinary sensitivity to initial conditions. 


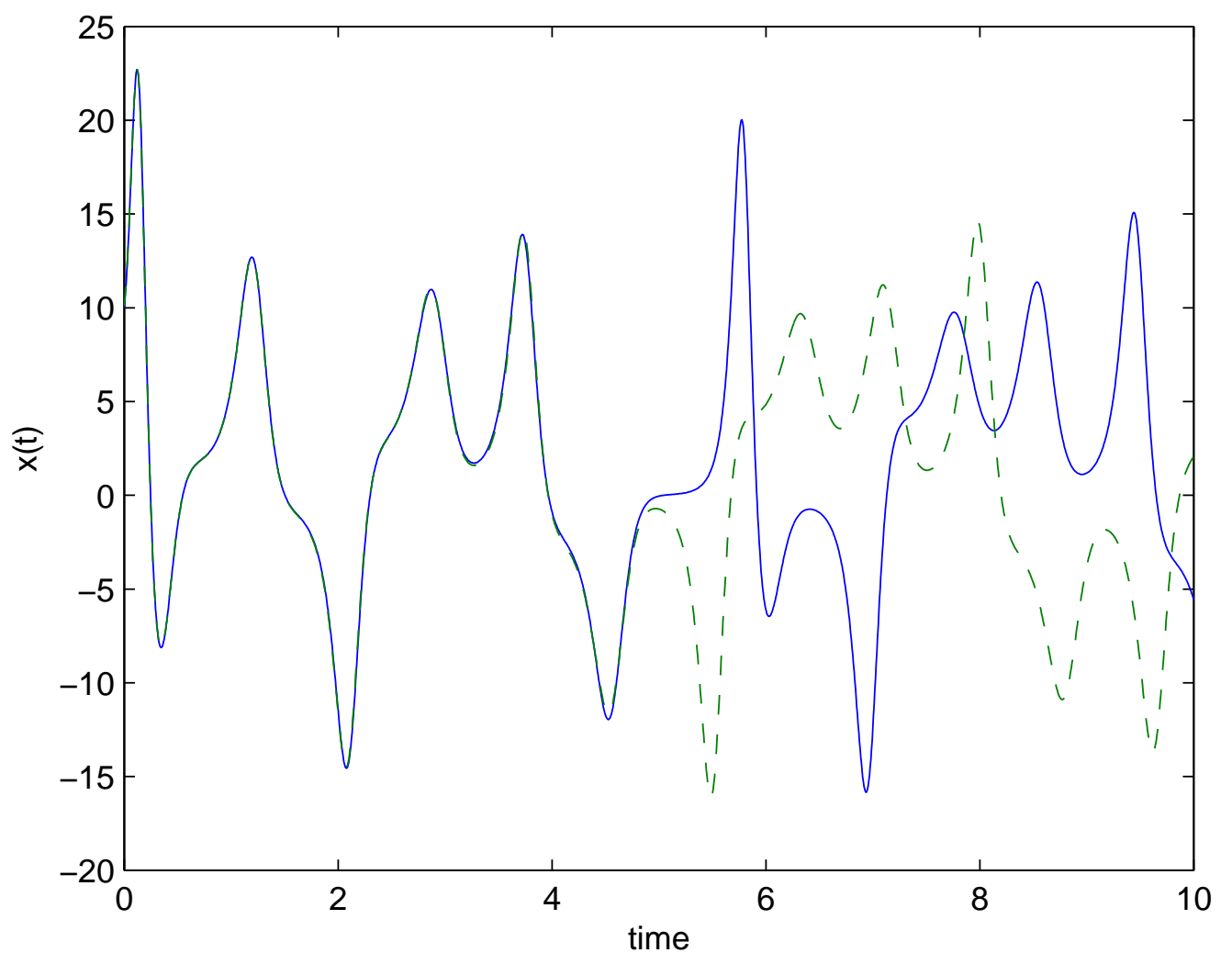

Figure 1.3: Divergence of two nearby Lorenz trajectories 


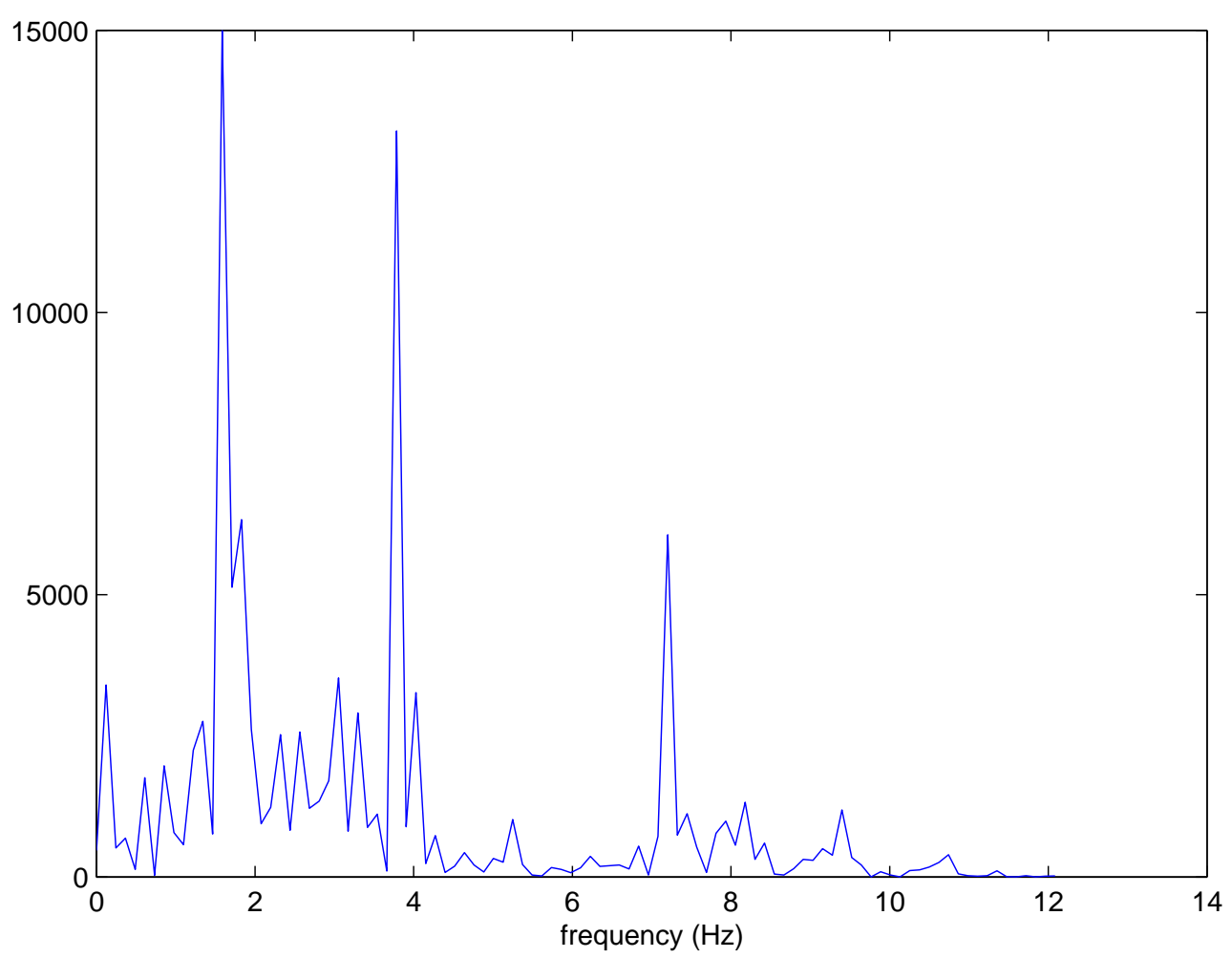

Figure 1.4: Power spectrum of $x(t)$ 


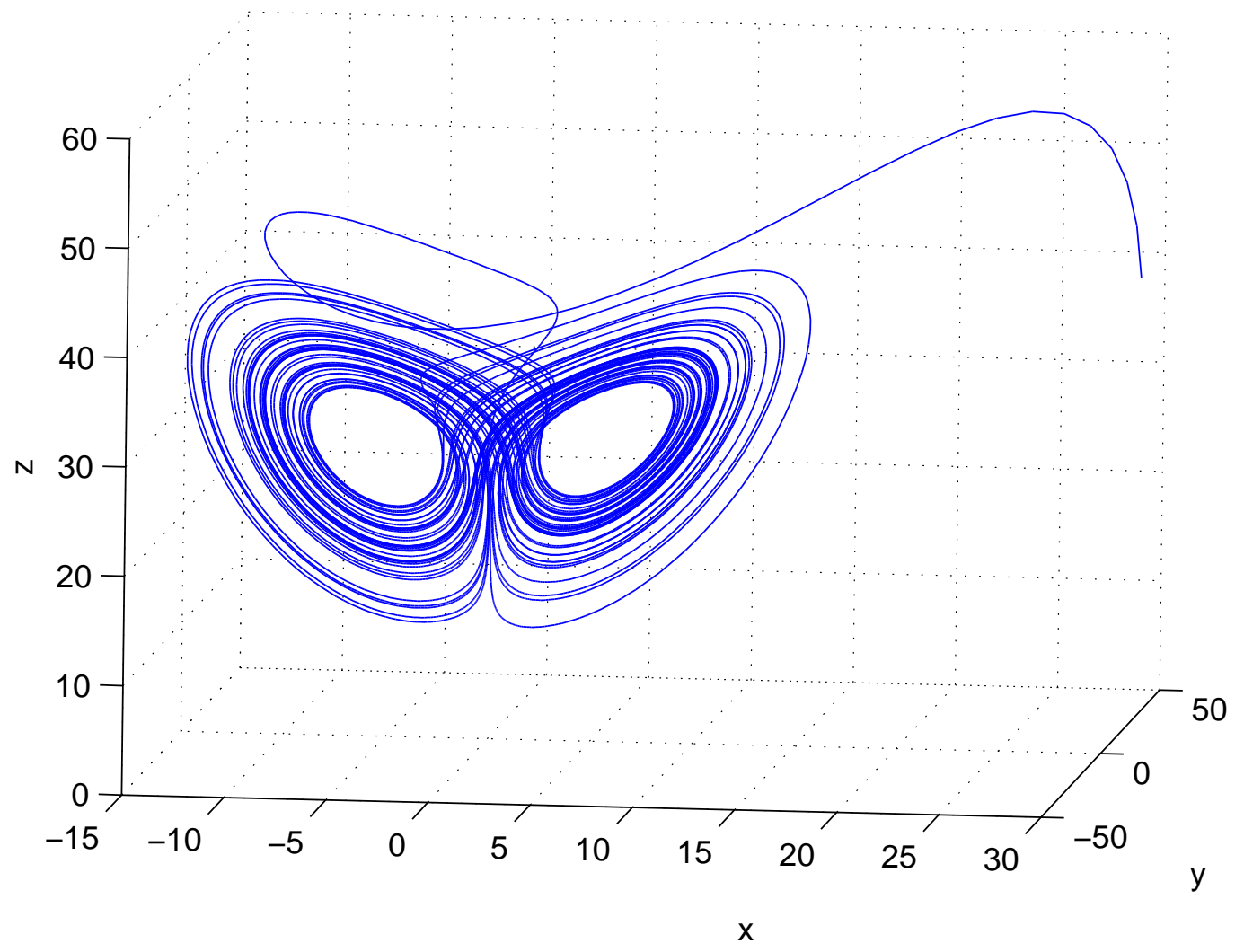

Figure 1.5: System trajectory trapped on a strange attractor 


\section{CHAPTER 2}

\section{THE LORENZ SYSTEM}

The Lorenz equations constitute one of the oldest known examples of a system that can undergo chaotic evolution in certain parameter regimes. The complex behavior it displays stands in stark contrast to its simple appearance, which is part of why the model has sparked a great deal of interest since its creation. It appears in a wide variety of contexts, including oscillations in a circuit [3], the behavior of a single-mode laser system [7], the operation of a disk dynamo [11], and in the dynamics of a chaotic waterwheel [21]. In this chapter we examine the behavior of the Lorenz model, locate its equilibrium points, and discuss the stability of solutions near those equilibrium points using a linear approximation to the system.

\subsection{The Lorenz Model}

The Lorenz model is a system of three coupled, first-order nonlinear differential equations,

$$
\left\{\begin{array}{l}
\dot{x}=p(y-x) \\
\dot{y}=R x-y-x z \\
\dot{z}=x y-b z,
\end{array}\right.
$$

where $p, R$, and $b$ are parameters discussed in the previous chapter. For the remainder

of this work, we will take $p=10$ and $b=1$, leaving $R$ as a free parameter. Despite the system's relatively simple appearance, there is no way to write down a solution of (2.1) in terms of either elementary or special functions. This is largely due to the presence of two nonlinear terms, $x z$ and $x y$. We must resort instead to numerical integration, for which we have many excellent algorithms to choose from. In this work, we use the classical fourth order Runge-Kutta method with a typical step size 
of $h=0.00625$, as described in appendix A. The solution to (2.1) consists of an infinite set of trajectories in $\mathbb{R}^{3}$ whose properties depend on the value of $R$. An individual trajectory is selected from this infinite set by choosing an initial condition.

Shown in Figures 2.1, 2.2, and 2.3 are both time series and phase space representations of the behavior of the Lorenz system for different values of $R$. For $R=0$ the system quickly reaches mechanical equilibrium as fluid flow ceases, leaving thermal equilibrium to be established by conduction. When $R=10$ the vertical temperature difference is large enough to drive convective motion, and the system quickly evolves to one of the two stable fixed points. For $R=28$ the two fixed points are unstable and the trajectory becomes trapped on a strange attractor. The system continuously hops from one nearly periodic orbit to the next, displaying the characteristic apparent unpredictability of a chaotic system. Notice the tightly coupled evolution of the fluid flow $x$ and the horizontal temperature difference $y$ present for both $R=10$ and $R=28$.
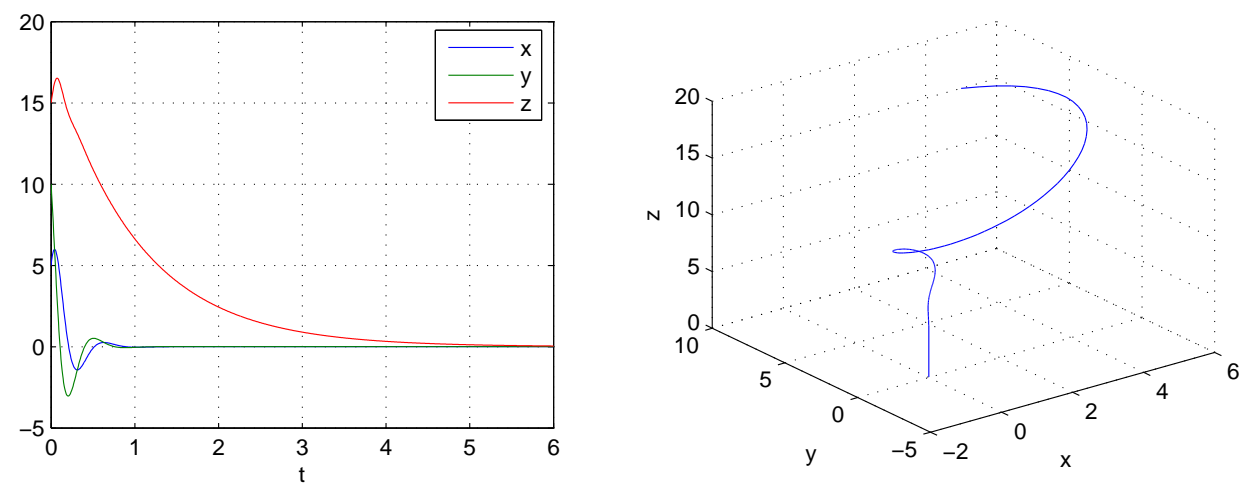

Figure 2.1: Lorenz System with $\mathrm{R}=0$

To get a better sense of how the overall behavior of the Lorenz system changes as $R$ increases, we can calculate how often the system visits any given value in the 

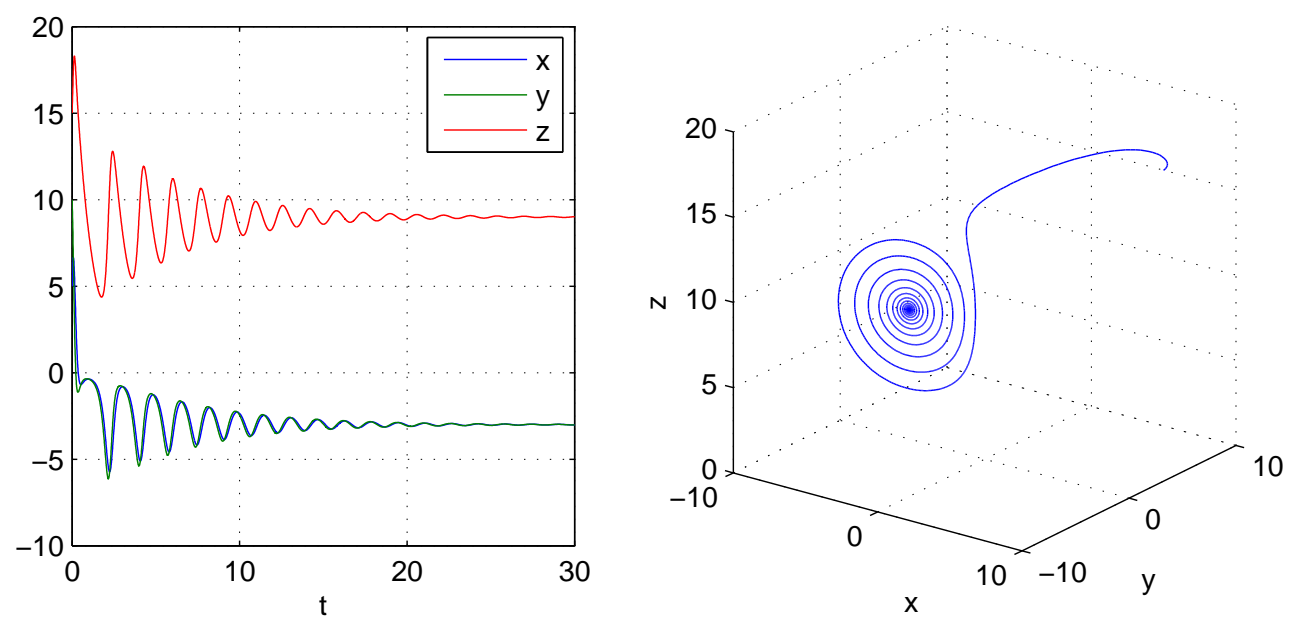

Figure 2.2: Lorenz System with $\mathrm{R}=10$
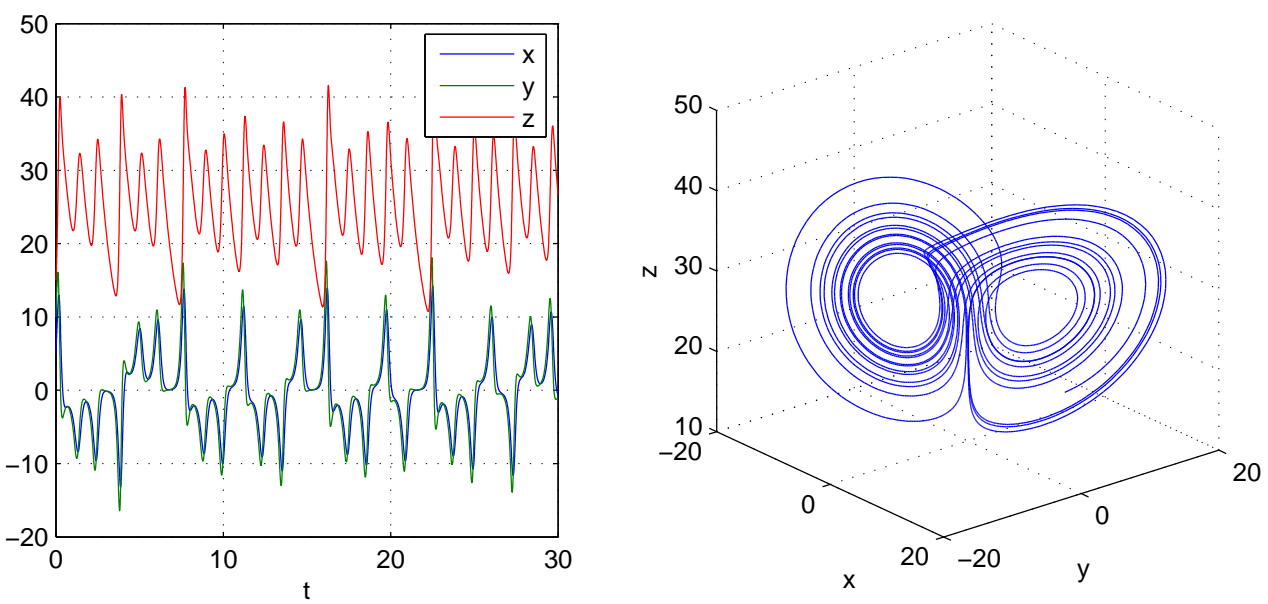

Figure 2.3: Lorenz System with $\mathrm{R}=28$ 
course of its evolution. For each value of $R$ we construct a histogram by dividing an interval of $x, y$, or $z$ into segments and counting how often the system falls into each bin. The results are shown in Figure 2.4 for $z$, where the darker the color the more often the system visits that value as it follows its trajectory. Plots constructed in this way are sometimes referred to as modified Feigenbaum diagrams. Figure 2.5 is a closer look at the range $0<R<15$, where the transition to chaotic behavior occurs. We see that as $R$ increases the equilibrium point moves further away from the origin and the system trajectories become more spread out in phase space.

\subsection{Equilibrium Points}

The point $\overline{\mathbf{x}} \in \mathbb{R}^{n}$ is an equilibrium point of the differential equation $\dot{\mathbf{x}}=\mathbf{f}(\mathbf{x})$ if it satisfies

$$
\mathbf{f}(\overline{\mathbf{x}})=\mathbf{0}
$$

for all $t$. For a system in $\mathbb{R}^{3}, \overline{\mathbf{x}}=(\bar{x}, \bar{y}, \bar{z})$ is an equilibrium point if it satisfies

$$
\dot{x}(\overline{\mathbf{x}})=\dot{y}(\overline{\mathbf{x}})=\dot{z}(\overline{\mathbf{x}})=0,
$$

for all $t$. From (2.1), we see that the equilibrium points of the Lorenz system must satisfy

$$
\begin{aligned}
10(\bar{y}-\bar{x}) & =0 \\
R \bar{x}-\bar{y}-\bar{x} \bar{z} & =0 \\
\bar{x} \bar{y}-\bar{z} & =0 .
\end{aligned}
$$

It is clear that the origin is an equilibrium point, so we will take $\overline{\mathbf{x}} \neq \mathbf{0}$ to look for others. From equation (2.2) we have $\bar{x}=\bar{y}$, such that equation (2.4) suggests $\bar{x}^{2}=\bar{z}$. 


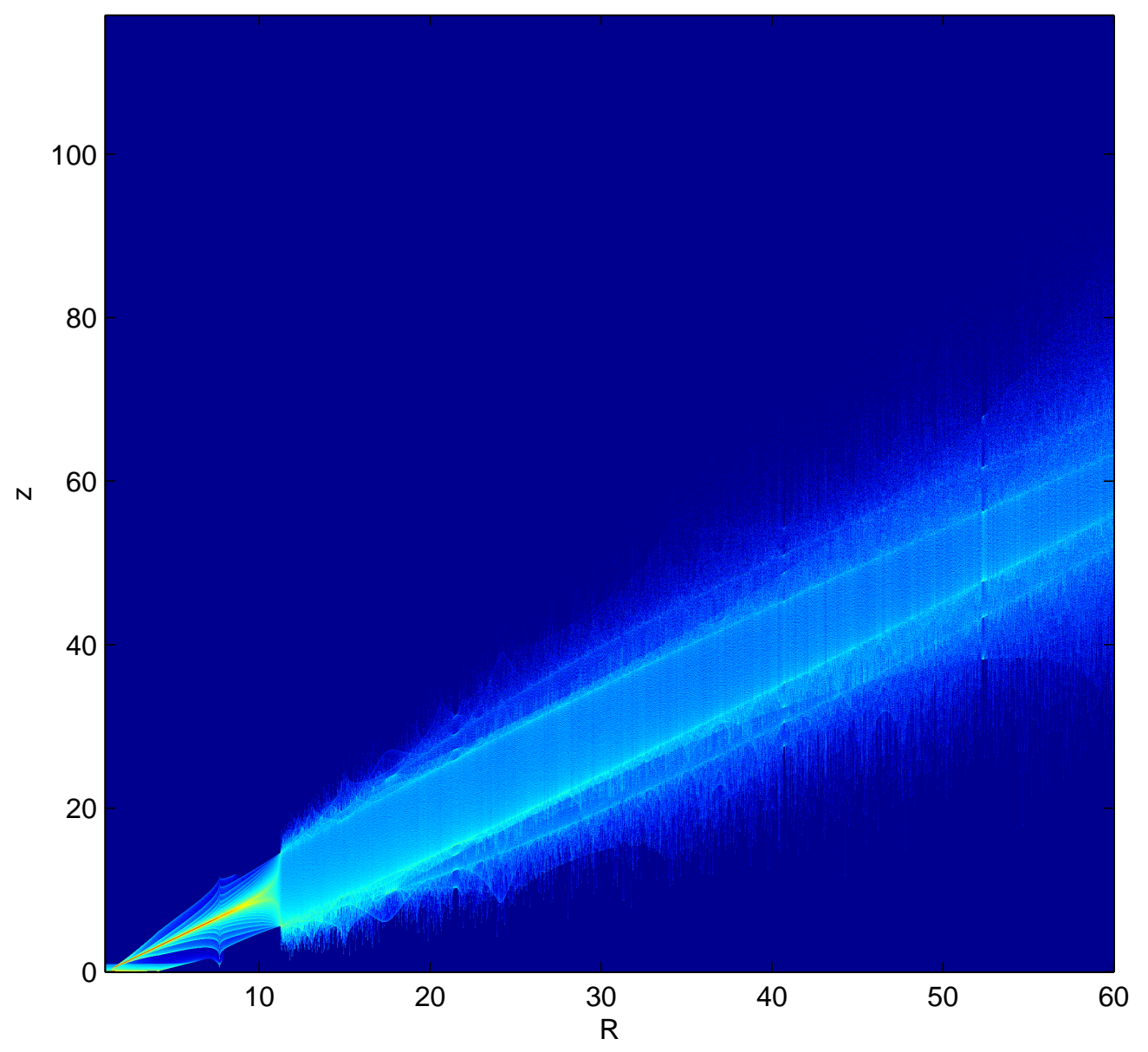

Figure 2.4: Behavior of the z-coordinate for $0<R<60$ 


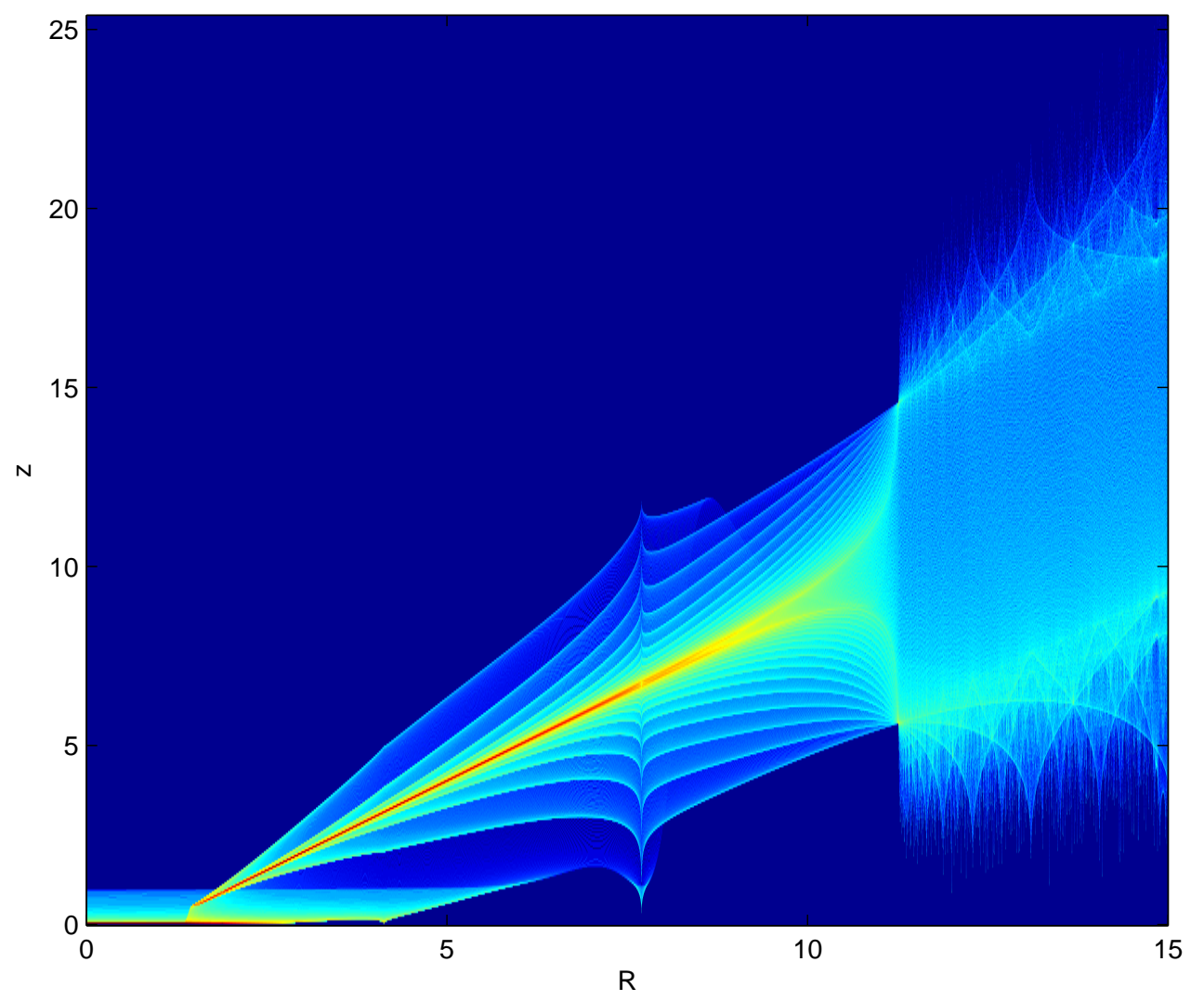

Figure 2.5: Behavior of the z-coordinate for $0<R<15$ 
Combining these expressions with $(2.3)$ gives $\bar{x}(R-1-\bar{z})=0$. If we take $\bar{x} \neq 0$, then $\bar{z}=R-1$ and $\bar{x}=\bar{y}= \pm \sqrt{R-1}$. As such, the locations of the equilibrium points are uniquely determined by the value of $\bar{x}$. For $R \leq 1$ the origin is the only equilibrium point. In this regime, conduction is sufficient to bring the system into equilibrium and there is no fluid motion in the steady state. For $R>1$, there are two additional equilibrium points which correspond to steady convective states - either clockwise or counterclockwise fluid flow at constant speed, occurring with equal probability if the system starts from a random initial state. Thus for $R>1$ there are three distinct equilibrium points located at

$$
\left\{\begin{array}{l}
\left(x_{0}, y_{0}, z_{0}\right)=(0,0,0) \\
\left(x_{1}, y_{1}, z_{1}\right)=(\sqrt{R-1}, \sqrt{R-1}, R-1) \\
\left(x_{2}, y_{2}, z_{2}\right)=(-\sqrt{R-1},-\sqrt{R-1}, R-1) .
\end{array}\right.
$$

If you place the system at an equilibrium point, it will stay there unless subjected to a perturbation. An equilibrium point $\overline{\mathbf{x}}$ is called asymptotically stable, or simply stable, if the system returns to $\overline{\mathbf{x}}$ after a perturbation is applied, while $\overline{\mathbf{x}}$ is called unstable if it does not return to $\overline{\mathbf{x}}$ after a perturbation is applied. In the following section we investigate the stability of these equilibrium points using a linear approximation. The following section contains elements adapted from [10].

\subsection{Linear Approximation near Equilibrium Points}

Consider the autonomous system of equations given by

$$
\left\{\begin{array}{l}
\dot{x}(x, y, z)=f(x, y, z) \\
\dot{y}(x, y, z)=g(x, y, z) \\
\dot{z}(x, y, z)=h(x, y, z)
\end{array}\right.
$$


where $x=x(t), y=y(t)$, and $z=z(t)$ are all functions of time. The set of points $\left\{\overline{\mathbf{x}}_{i}\right\}$ where $f\left(\overline{\mathbf{x}}_{i}\right)=g\left(\overline{\mathbf{x}}_{i}\right)=h\left(\overline{\mathbf{x}}_{i}\right)=0$ for all time $t$ is the set of equilibrium points of the system. In order to discern the stability properties of the system near its equilibrium points, we will consider a linear approximation to (2.6) near those points. Geometrically, we are approximating a complicated surface in phase space by a hyperplane an approximation that will only be valid in a neighborhood around each equilibrium point. Using Taylor's theorem, we can expand the system (2.6) about one of the equilibrium points $\overline{\mathbf{x}}=(\bar{x}, \bar{y}, \bar{z})$. Keeping only first order terms, we get

$$
\begin{aligned}
& \dot{x}(x, y, z)=\dot{x}(\bar{x}, \bar{y}, \bar{z})+\left.(x-\bar{x}) \frac{\partial f}{\partial x}\right|_{\mathbf{x}=\overline{\mathbf{x}}}+\left.(y-\bar{y}) \frac{\partial f}{\partial y}\right|_{\mathbf{x}=\overline{\mathbf{x}}}+\left.(z-\bar{z}) \frac{\partial f}{\partial z}\right|_{\mathbf{x}=\overline{\mathbf{x}}}+\ldots \\
& \dot{y}(x, y, z)=\dot{y}(\bar{x}, \bar{y}, \bar{z})+\left.(x-\bar{x}) \frac{\partial g}{\partial x}\right|_{\mathbf{x}=\overline{\mathbf{x}}}+\left.(y-\bar{y}) \frac{\partial g}{\partial y}\right|_{\mathbf{x}=\overline{\mathbf{x}}}+\left.(z-\bar{z}) \frac{\partial g}{\partial z}\right|_{\mathbf{x}=\overline{\mathbf{x}}}+\ldots \\
& \dot{z}(x, y, z)=\dot{z}(\bar{x}, \bar{y}, \bar{z})+\left.(x-\bar{x}) \frac{\partial h}{\partial x}\right|_{\mathbf{x}=\overline{\mathbf{x}}}+\left.(y-\bar{y}) \frac{\partial h}{\partial y}\right|_{\mathbf{x}=\overline{\mathbf{x}}}+\left.(z-\bar{z}) \frac{\partial h}{\partial z}\right|_{\mathbf{x}=\overline{\mathbf{x}}}+\ldots
\end{aligned}
$$

where $\dot{x}(\bar{x}, \bar{y}, \bar{z})=\dot{y}(\bar{x}, \bar{y}, \bar{z})=\dot{z}(\bar{x}, \bar{y}, \bar{z})=0$. This can be expressed in matrix form as

$$
\left(\begin{array}{c}
\dot{x} \\
\dot{y} \\
\dot{z}
\end{array}\right)=\left(\begin{array}{ccc}
\frac{\partial f}{\partial x} & \frac{\partial f}{\partial y} & \frac{\partial f}{\partial z} \\
\frac{\partial g}{\partial x} & \frac{\partial g}{\partial y} & \frac{\partial g}{\partial z} \\
\frac{\partial h}{\partial x} & \frac{\partial h}{\partial y} & \frac{\partial h}{\partial z}
\end{array}\right)_{\mathbf{x}=\overline{\mathbf{x}}}\left(\begin{array}{c}
x-\bar{x} \\
y-\bar{y} \\
z-\bar{z}
\end{array}\right)
$$

or in more compact notation,

$$
\dot{\mathbf{x}}=J(\overline{\mathbf{x}})(\mathbf{x}-\overline{\mathbf{x}})
$$

where $J(\overline{\mathbf{x}})$ is the Jacobian of the system evaluated at one of the equilibrium points. We look for solutions of the form

$$
\mathbf{x}=\overline{\mathbf{x}}+\mathbf{r} e^{\lambda t}
$$

where $\lambda$ is a constant. In order to satisfy (2.7), we must have

$$
J \mathbf{r} e^{\lambda t}-\lambda \mathbf{r} e^{\lambda t}=(J-\lambda I) \mathbf{r} e^{\lambda t}=\mathbf{0}
$$


for all $t$, or since $e^{\lambda t}$ is nonzero for all finite $t$,

$$
(J-\lambda I) \mathbf{r}=\mathbf{0} .
$$

Given a value for $\lambda$, this is equivalent to three linear equations for the components of r. It is known from elementary linear algebra that a system of homogeneous linear equations has non-trivial solutions if and only if

$$
\operatorname{det}(J-\lambda I)=0
$$

In component form this becomes

$$
\left|\begin{array}{ccc}
j_{11}-\lambda & j_{12} & j_{13} \\
j_{21} & j_{22}-\lambda & j_{23} \\
j_{31} & j_{32} & j_{33}-\lambda
\end{array}\right|=0
$$

where $j_{i j}$ are the entries of $J$. This equation is a third-degree polynomial in $\lambda$, called the characteristic equation. By the fundamental theorem of algebra, a third-degree polynomial such as this one has three roots, real or complex, some of which may be repeated roots [10].

Suppose that there are exactly three distinct eigenvalues $\lambda_{1}, \lambda_{2}$, and $\lambda_{3}$, which may be real, complex, or zero. For each $\lambda_{i}$ there exist non-zero solutions $\mathbf{r}=\mathbf{r}_{\mathbf{i}}$ of the equation (2.7), called eigenvectors. The general solution of equation (2.7) is given by a linear combination of the eigenvectors of the system, written as

$$
\mathbf{x}=\overline{\mathbf{x}}+c_{1} \mathbf{r}_{1} e^{\lambda_{1} t}+c_{2} \mathbf{r}_{2} e^{\lambda_{2} t}+c_{3} \mathbf{r}_{\mathbf{3}} e^{\lambda_{3} t}
$$

where $c_{1}, c_{2}$ and $c_{3}$ are constants. Clearly, the stability of the system near an equilibrium point depends on whether the eigenvalues of the Jacobian $J$ at that point are positive, negative, zero, or complex. For example, if the eigenvalues are all negative 
and real, the solution (2.8) will converge to $\overline{\mathbf{x}}$ at $t$ approaches infinity and we say that the system is stable near that point. However, if one or more of the eigenvalues has a positive real part, the solution (2.8) will diverge to infinity along the direction(s) of the associated eigenvector(s). Finally, if one or more of the eigenvalues has an imaginary part, the solution $\mathbf{x}$ will oscillate along the direction(s) of the associated eigenvector(s), though the equilibrium point may still be stable if the real parts of the eigenvalues are all negative. The question of the stability of (2.1) near an equilibrium point is thus reduced to a study of the locations on the complex plane of the eigenvalues of the Jacobian at that point.

\subsection{Stability near Equilibrium Points}

If all of the eigenvalues $\lambda_{i}$ of the Jacobian $J(\overline{\mathbf{x}})$ (which are also called the roots of the characteristic equation) are in the left half-plane, then the equilibrium point at which the Jacobian is evaluated is stable. A necessary and sufficient condition that all roots $\lambda_{i}$ of the characteristic equation lie in the left half-plane can be found in Routh's criterion, which we present here as a theorem,

Theorem 2.4.1. All the roots of the real polynomial $P(z)$ have negative real parts if and only if in the carrying out of Routh's Algorithm all the elements of the first column of Routh's scheme are different from zero and of like sign.

A proof can be found in [6]. To get a better sense of Routh's Algorithm, consider the third-degree polynomial

$$
P(x)=a_{3} x^{3}+a_{2} x^{2}+a_{1} x^{1}+a_{0}
$$

The Routh-Hurwitz test involves arranging the coefficients of the characteristic poly- 
nomial in a certain way (Routh's Algorithm) and counting the number of sign changes in the first column. For a third-order polynomial such as (2.9), the Routh array is

$$
\begin{array}{|c|cc}
x^{3} & a_{3} & a_{1} \\
x^{2} & a_{2} & a_{0} \\
x^{1} & c & 0 \\
x^{0} & d & 0
\end{array},
$$

where

$$
c=\frac{a_{1} a_{2}-a_{0} a_{3}}{a_{2}}
$$

and $d=a_{0}$. The number of distinct right half-plane (RHP) roots of the polynomial $P(x)$ is equal to the number of algebraic sign changes in the leftmost column of coefficients, read from top to bottom. The number of distinct left half-plane (LHP) roots can be determined by counting the number of sign changes in the left column of the Routh array of $P(-x)$. Since a polynomial has a number of roots equal to its degree $n$, we can say

$$
n=n_{R}+n_{L}+n_{I},
$$

where $n_{R}$ is the number of RHP roots, $n_{L}$ is the number of LHP roots, and $n_{I}$ is the number of imaginary axis roots, which can be deduced by finding $n_{R}$ and $n_{L}$ first. However, for questions of stability it is easier to apply Theorem 2.4.1 to the system under consideration. Several practical examples are worked out in [20].

We can now apply Theorem 2.4.1 to the equilibrium points of the Lorenz system. Our system of coupled first-order differential equations is

$$
\left\{\begin{aligned}
\dot{x} & =10(y-x) \\
\dot{y} & =R x-y-x z \\
\dot{z} & =x y-z,
\end{aligned}\right.
$$


with a Jacobian matrix

$$
J(x, y, z)=\left(\begin{array}{ccc}
-10 & 10 & 0 \\
R-z & -1 & -x \\
y & x & -1
\end{array}\right) .
$$

The characteristic polynomial $P(\lambda)$ of $J(x, y, z)$ is

$$
P(\lambda)=\left|\begin{array}{ccc}
-10-\lambda & 10 & 0 \\
R-z & -1-\lambda & -x \\
y & x & -1-\lambda
\end{array}\right|
$$

or equivalently,

$$
P(\lambda)=-\lambda^{3}-12 \lambda^{2}-\left(21+x^{2}+10 z-10 R\right) \lambda-10\left(1+x^{2}+x y+z-R\right) .
$$

We do not so much care about the exact values of the eigenvalues $\lambda$ as we do their location in the complex plane, which can be determined using a Routh array. The coefficients of $P(\lambda)$ are

$$
\left\{\begin{array}{l}
a_{3}=-1 \\
a_{2}=-12 \\
a_{1}=-\left(21+x^{2}+10 z-10 R\right) \\
a_{0}=-10\left(1+x^{2}+x y+z-R\right),
\end{array}\right.
$$

So the first column of the Routh array associated with $P(\lambda)$ at the origin $\left(x_{0}, y_{0}, z_{0}\right)=$ $(0,0,0)$ is

$$
\begin{array}{l|l}
\lambda^{3} & -1 \\
\lambda^{2} & -12 \\
\lambda^{1} & -\frac{1}{6}(121-55 R) \\
\lambda^{0} & -10(1-R)
\end{array}
$$


For $R<1$, there are zero sign changes in the first column, so there are no roots located in the RHP and the system is stable at that point by Theorem 2.4.1. As $R$ crosses one, the number of sign changes in the first column increases from zero to one and the state of rest (the origin) becomes unstable. This is confirmed numerically in Figure 2.6, for which the system was placed very close to the origin (within a radius of 0.01) and allowed to evolve until $t_{f}=30$. As in Figure 2.4, darker colors correspond to values of $x$ or $z$ that the system visited more often in its evolution. Note the emergence of a stable equilibrium point away from the origin for $R>1$ in (2.6).

Now consider one of the equilibrium points that appear when $R>1$, specifically $\left(x_{1}, y_{1}, z_{1}\right)=(\sqrt{R-1}, \sqrt{R-1}, R-1)$. The coefficients of $P(\lambda)$ evaluated at $\left(x_{1}, y_{1}, z_{1}\right)$ are

$$
\left\{\begin{array}{l}
a_{3}=-1 \\
a_{2}=-12 \\
a_{1}=-(10+R) \\
a_{0}=-20(R-1),
\end{array}\right.
$$

so the Routh table associated with $P(\lambda)$ is

$$
\mid \begin{array}{l|l}
\lambda^{3} & -1 \\
\lambda^{2} & -12 \\
\lambda^{1} & -\frac{1}{3}(35-2 R) \\
\lambda^{0} & -20(R-1)
\end{array}
$$

For $1<R<17.5$ there are no sign changes in the first column of the Routh array and all the entries are different from zero, so by Theorem $2.4 .1,\left(x_{1}, y_{1}, z_{1}\right)$ is a stable equilibrium point in that parameter regime. For $R>17.5$ the number of roots in the LHP drops from three to one as two of the roots move into the RHP, causing $\left(x_{1}, y_{1}, z_{1}\right)$ to become unstable. This transition from stability to instability can be 


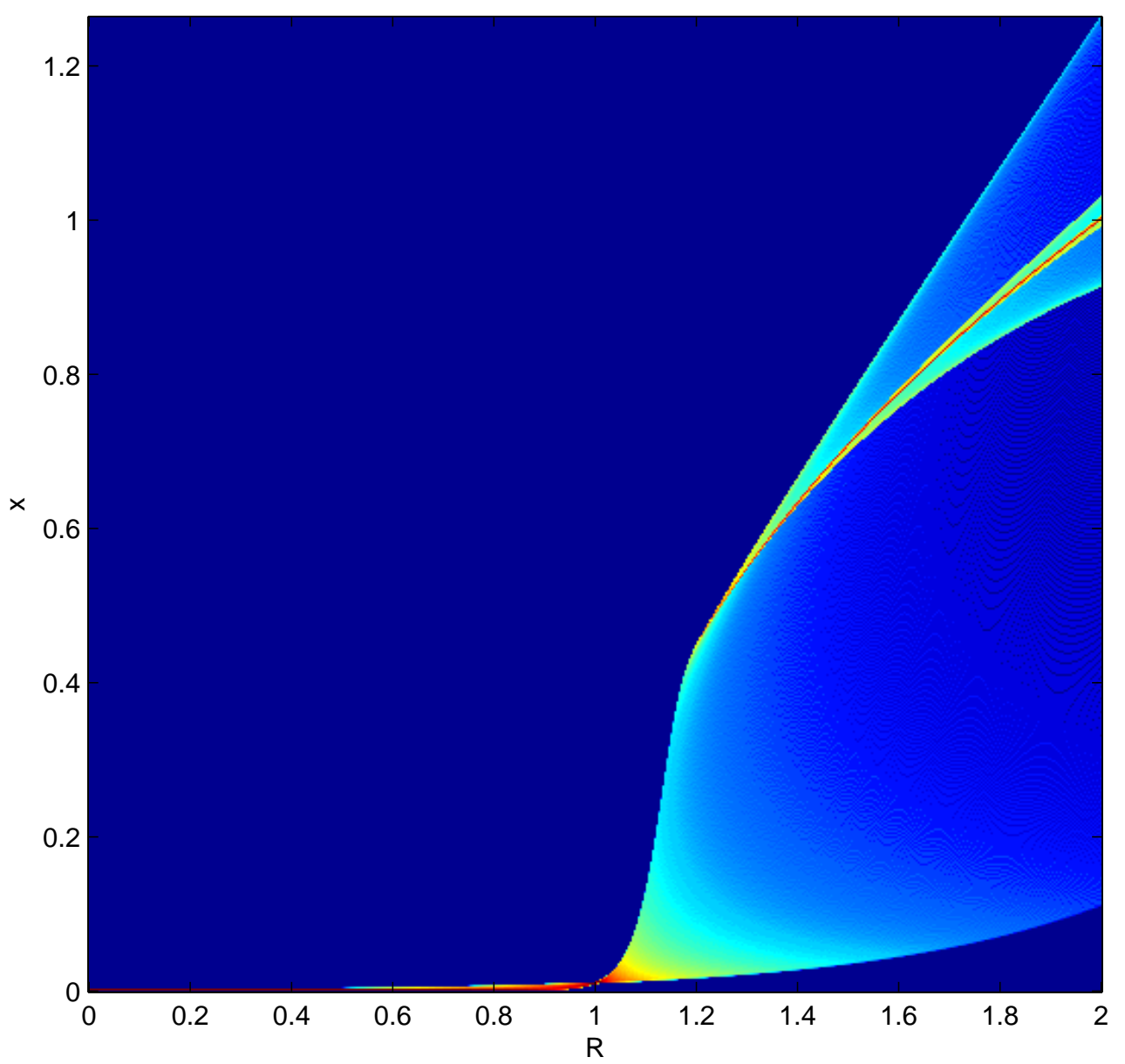

Figure 2.6: Stability of origin measured by $x$ 
seen in Figures 2.7 and 2.8, in which the system is placed close to the equilibrium point $\left(x_{1}, y_{1}, z_{1}\right)$ and allowed to evolve for $t_{f}=30$. A comparison of Figures 2.4 and 2.7 reveals that the appearance of the strange attractor actually occurs before the equilibrium points become unstable.

Since the coefficients of $P(\lambda)$ are even under the transformation $(x, y, z) \rightarrow$ $(-x,-y, z)$, the stability properties of $\left(x_{2}, y_{2}, z_{2}\right)=(-\sqrt{R-1},-\sqrt{R-1}, R-1)$ are identical to those of $\left(x_{1}, y_{1}, z_{1}\right)=(\sqrt{R-1}, \sqrt{R-1}, R-1)$. To summarize, the three equilibrium points under consideration are listed below with the parameter regimes in which they are stable and unstable.

$$
\begin{array}{lll} 
& \text { Stable } & \text { Unstable } \\
\left(x_{0}, y_{0}, z_{0}\right)=(0,0,0) & R \leq 1 & R>1 \\
\left(x_{1}, y_{1}, z_{1}\right)=(\sqrt{R-1}, \sqrt{R-1}, R-1) & 1<R<17.5 & R>17.5 \\
\left(x_{2}, y_{2}, z_{2}\right)=(-\sqrt{R-1},-\sqrt{R-1}, R-1) & 1<R<17.5 & R>17.5 .
\end{array}
$$

Note that for the case $R=17.5$, a zero appears in the first column of the Routh table, which indicates an imaginary axis root and therefore a possible periodic orbit. This orbit can be seen in Figure 2.9, which is the system response at $R=17.5$ when the system begins near one of the equilibrium points.

In the next chapter, we shall address the question of stabilizing the chaotic behavior of the Lorenz system using a feedback loop. 


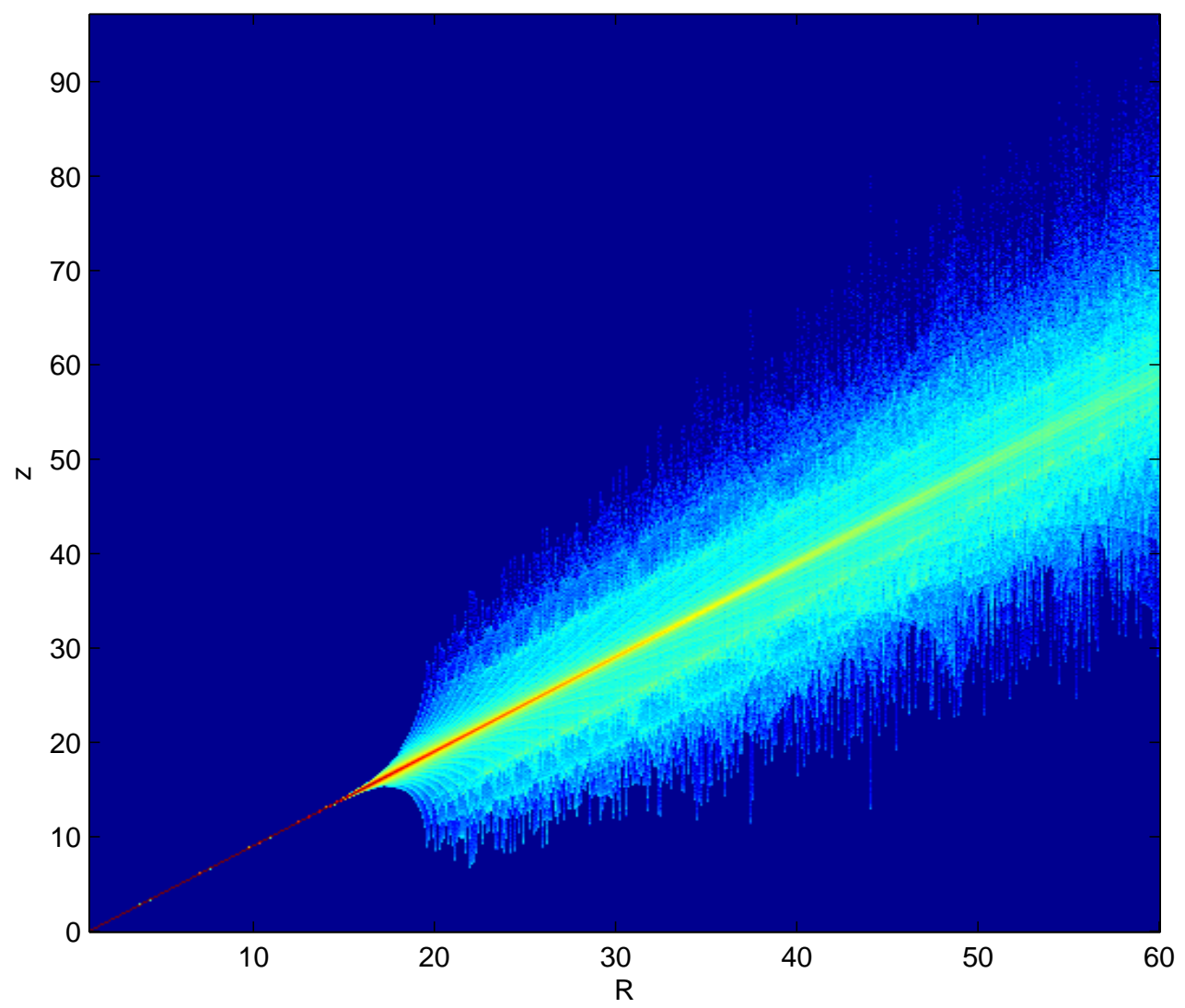

Figure 2.7: Stability of equilibrium point $\left(x_{1}, y_{1}, z_{1}\right)$ measured by $z$ 


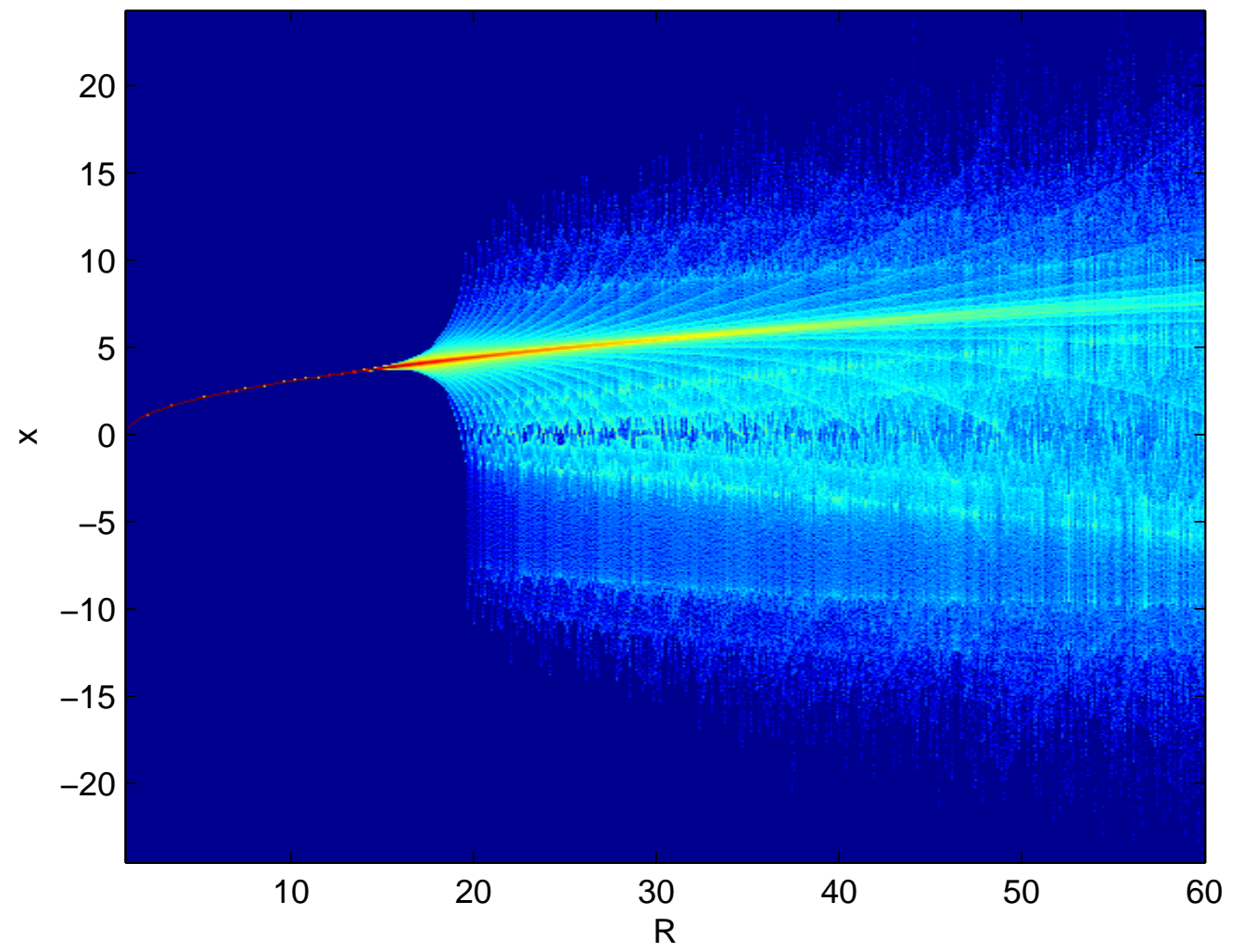

Figure 2.8: Stability of equilibrium point $\left(x_{1}, y_{1}, z_{1}\right)$ measured by $x$ 

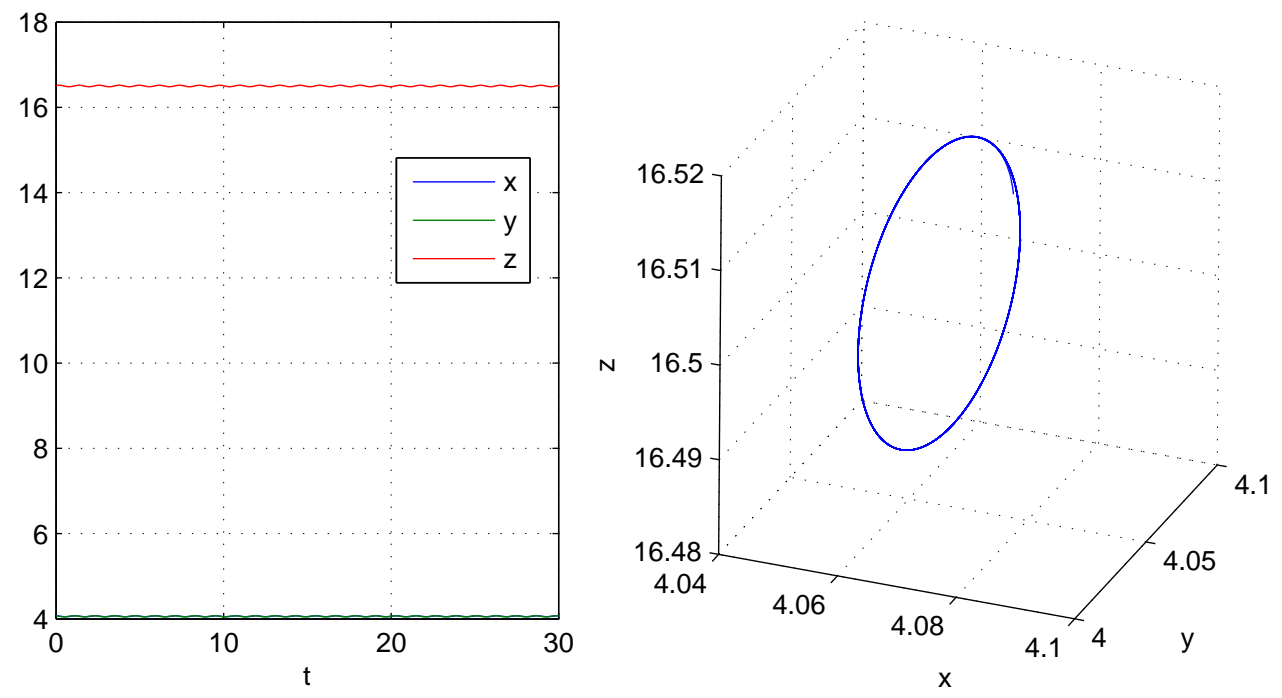

Figure 2.9: Periodic orbit for $R=17.5$ 


\section{CHAPTER 3}

\section{THE CONTROLLED LORENZ SYSTEM}

Many authors have proposed control schemes for chaotic systems. In reference [16], it is shown that a chaotic attractor can be converted into a periodic one using small time-dependent perturbations of a system parameter. The authors of reference [18] also achieve the stabilization of unstable periodic orbits of a chaotic system using a specially-designed external oscillator. Some of these control schemes have been implemented successfully in experiments - one such experiment involving a magnetoelastic ribbon is discussed in reference [4]. In another experiment a perturbative feedback scheme was used to suppress chaotic behavior in a thermal convection loop [19].

In this chapter we show that the strange attractor associated with the Lorenz system can be converted into a locally stable fixed point using a continuous statefeedback controller that involves a quotient of system parameters. As in the previous chapter, we will make use of a linear approximation and Routh-Hurwitz testing to evaluate the stability of the system near its equilibrium points.

\subsection{Quotient Controller}

By making adjustments to the parameter $R$ in response to the measured properties of the system, we establish a feedback loop that can be used to suppress chaotic behavior. The equations of the controlled Lorenz system are

$$
\left\{\begin{aligned}
\dot{x} & =10(y-x) \\
\dot{y} & =(R+u) x-y-x z \\
\dot{z} & =x y-z,
\end{aligned}\right.
$$


where the controller $u$ is given by the quotient

$$
u=-k \frac{y}{z}
$$

with a feedback gain $k$ and $z \neq 0$. One design advantage of this controller is that both $y$ and $z$ are easy to measure, since they are both proportional to temperature differences between different segments of the thermosyphon. In Figures 3.1, 3.2, and 3.3, the behavior of the Lorenz system under the influence of the feedback controller $u$ is shown, where the controller is activated at time $t=25$, halfway through each simulation. Clearly, the controller is capable of stabilizing the Lorenz system well into its normally chaotic regime.
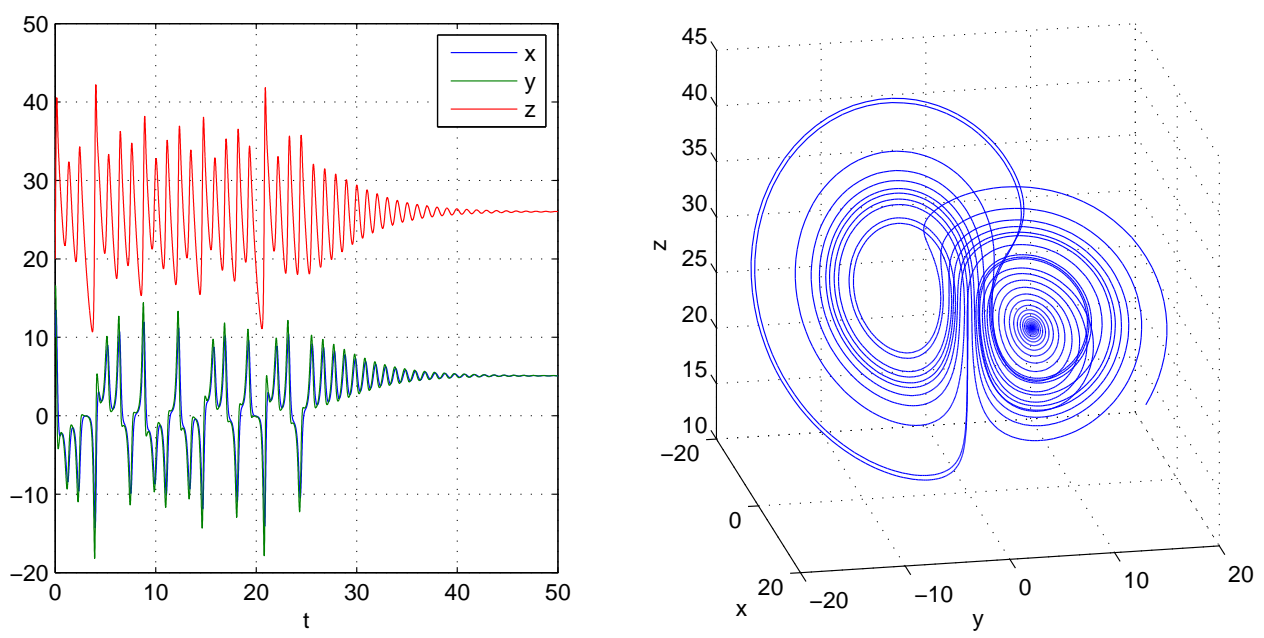

Figure 3.1: Controlled Lorenz system for $R=28$ and $k=5$ 

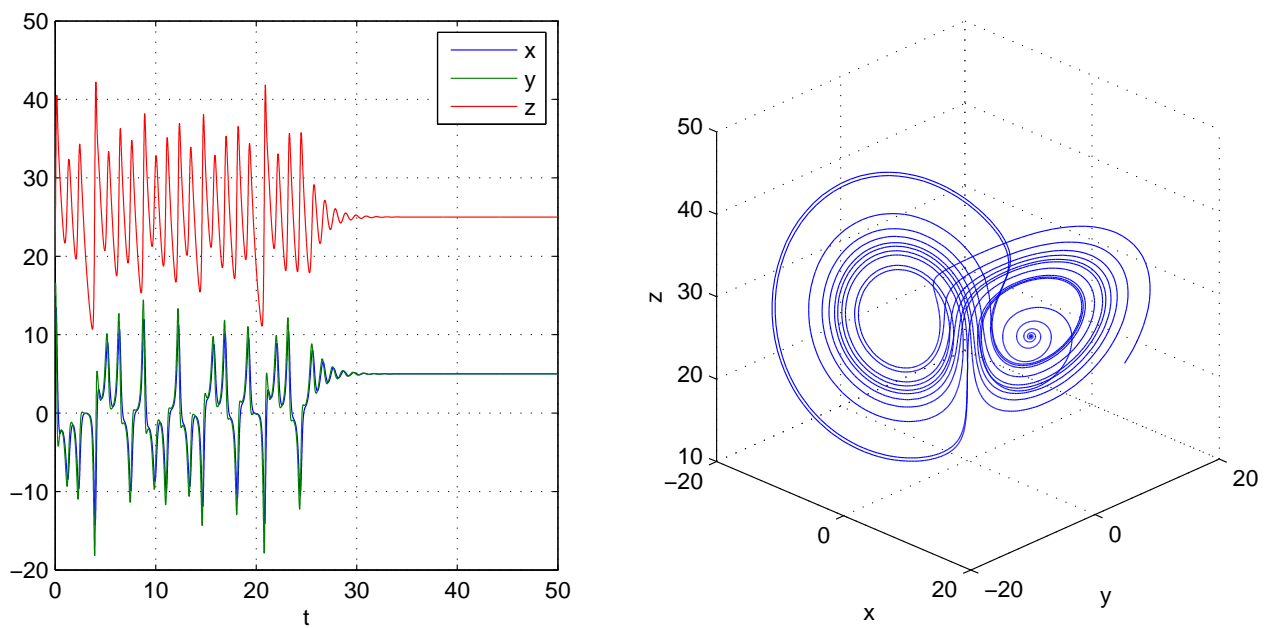

Figure 3.2: Controlled Lorenz system for $R=28$ and $k=10$
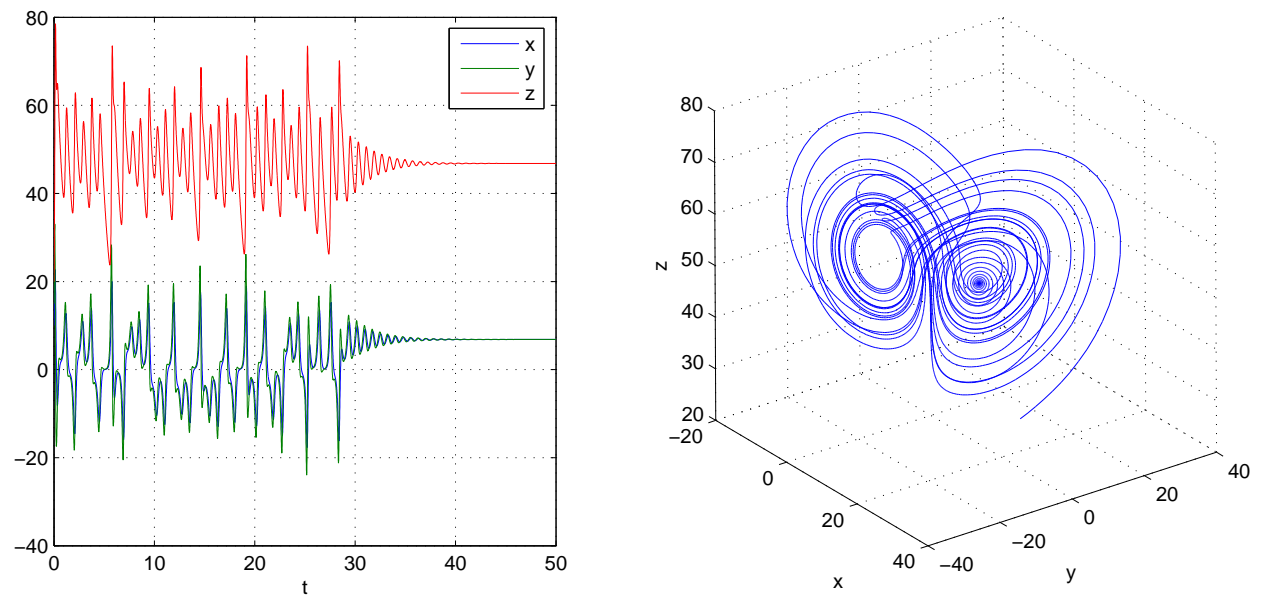

Figure 3.3: Controlled Lorenz system for $R=50$ and $k=15$ 


\subsection{Equilibrium Points}

The equilibrium points of (3.1) must satisfy $\dot{x}=\dot{y}=\dot{z}=0$, or

$$
\left\{\begin{aligned}
10(\bar{y}-\bar{x}) & =0 \\
\left(R-k \frac{\bar{y}}{\bar{z}}\right) \bar{x}-\bar{y}-\bar{x} \bar{z} & =0 \\
\bar{x} \bar{y}-\bar{z} & =0
\end{aligned}\right.
$$

The first and third expressions give us $\bar{x}=\bar{y}$ and $\bar{z}=\bar{x}^{2}$, which we can substitute into the second equation for

$$
\bar{x}\left[\left(R-k \bar{x}^{-1}\right)-\bar{x}^{2}-1\right]=0,
$$

which is equivalent to the depressed cubic

$$
\bar{x}^{3}-(R-1) \bar{x}+k=0 .
$$

The locations of the equilibrium points of (3.1) are uniquely determined by the roots of the cubic (3.4), which will clearly depend on the values of $k$ and $R$. Let $G \subset \mathbb{R}^{2}$ be our parameter space defined by

$$
G=\left\{(R, k) \mid R_{\min } \leq R \leq R_{\max }, k_{\min } \leq k \leq k_{\max }\right\}
$$

where we will let $R_{\min }=24, R_{\max }=60, k_{\min }=0$, and $k_{\max }=20$.

In order to solve the cubic (3.4) we can use techniques presented in reference [9]. The character of the roots of a depressed cubic $x^{3}+p x+q$ can be determined by the value taken by the polynomial discriminant $D$,

$$
D=\left(\frac{p}{3}\right)^{3}+\left(\frac{q}{2}\right)^{2}
$$

The solutions of the depressed cubic can then be classified as follows, 
1. $D>0$; one real and two complex solutions,

2. $D=0$; three real solutions including a double solution,

3. $D<0$; three real distinct solutions.

For equation (3.4), $D=\frac{1}{4} k^{2}-\frac{1}{27}(R-1)^{3}$. Since $D<0$ for all $(R, k) \in G$, equation (3.4) has three real, distinct solutions which can be written as

$$
\left\{\begin{array}{l}
x_{1}=2 \sqrt{\frac{|R-1|}{3}} \cos \left(\frac{\phi}{3}\right) \\
x_{2}=-2 \sqrt{\frac{|R-1|}{3}} \cos \left(\frac{\phi-\pi}{3}\right) \\
x_{3}=-2 \sqrt{\frac{|R-1|}{3}} \cos \left(\frac{\phi+\pi}{3}\right)
\end{array}\right.
$$

where $\phi$ can be calculated using

$$
\cos (\phi)=\frac{-k}{2 \sqrt{\left(\frac{|R-1|}{3}\right)^{3}}}
$$

Since $k>0$, we can always express $\phi$ in the range $\frac{\pi}{2}<\phi<\pi$ without loss of generality, which allows us to place bounds on the locations of the roots $x_{1}, x_{2}$, and $x_{3}$. Since $\frac{\pi}{2}<\phi<\pi$, we have $\frac{\pi}{6}<\frac{\phi}{3}<\frac{\pi}{3}$ and thus $\frac{1}{2}<\cos \left(\frac{\phi}{3}\right)<\frac{\sqrt{3}}{2}$. This implies that the root $x_{1}$ must be in the range

$$
\sqrt{\frac{|R-1|}{3}}<x_{1}<\sqrt{|R-1|} .
$$

Similarly, we can place bounds on the location of $x_{2}$. Since $\frac{\sqrt{3}}{2}<\cos \left(\frac{\phi-\pi}{3}\right)<1$, we have

$$
-2 \sqrt{\frac{|R-1|}{3}}<x_{2}<-\sqrt{|R-1|}
$$

Finally, since $-\frac{1}{2}<\cos \left(\frac{\phi+\pi}{3}\right)<0$, the root $x_{3}$ is in the range

$$
0<x_{3}<\sqrt{\frac{|R-1|}{3}} .
$$


Interestingly, the ranges in which the roots can be found do not depend on the value of $k$, though the locations of the equilibrium points depend on both $R$ and $k$. As we will see in the next section, the stability of the system near its equilibrium points also depends on both $R$ and $k$.

\subsection{Local Stability of the Controlled Lorenz System}

The Jacobian matrix of the controlled Lorenz system (3.1) is

$$
J(x, y, z)=\left(\begin{array}{ccc}
-10 & 10 & 0 \\
R-k \frac{y}{z}-z & -k \frac{x}{z}-1 & k \frac{x y}{z^{2}}-x \\
y & x & -1
\end{array}\right) .
$$

Evaluated at the equilibrium point $x_{0}$, which could be $x_{1}, x_{2}$, or $x_{3}$, we have

$$
J\left(x_{0}, y_{0}, z_{0}\right)=\left(\begin{array}{ccc}
-10 & 10 & 0 \\
R-\frac{k}{x_{0}}-x_{0}^{2} & -\frac{k}{x_{0}}-1 & \frac{k}{x_{0}^{2}}-x_{0} \\
x_{0} & x_{0} & -1
\end{array}\right),
$$

since $x_{0}=y_{0}$ and $z_{0}=x_{0}^{2}$. The characteristic equation $P(\lambda)$ for the Jacobian is given by

$$
P(\lambda)=\left|\begin{array}{ccc}
-10-\lambda & 10 & 0 \\
R-\frac{k}{x_{0}}-x_{0}^{2} & -\frac{k}{x_{0}}-1-\lambda & \frac{k}{x_{0}^{2}}-x_{0} \\
x_{0} & x_{0} & -1-\lambda
\end{array}\right|=0
$$

or equivalently by

$$
\lambda^{3}+\left(12+\frac{k}{x_{0}}\right) \lambda^{2}+\left(20 \frac{k}{x_{0}}+11 x_{0}^{2}-10 R+21\right) \lambda+\left(10-10 R+30 x_{0}^{2}\right)=0
$$


We will refer to the coefficients as

$$
\left\{\begin{array}{l}
a=12+\frac{k}{x_{0}} \\
b=20 \frac{k}{x_{0}}+11 x_{0}^{2}-10 R+21 \\
c=10-10 R+30 x_{0}^{2}
\end{array}\right.
$$

A necessary and sufficient condition for an equilibrium point $x_{0}$ to be locally stable is that all eigenvalues $\lambda$ of the characteristic polynomial lie in the left half-plane. Since the full expressions for the solutions of (3.10) are quite unwieldy and we only need to determine the locations of the roots in the complex plane, we will again employ Routh-Hurwitz testing. As in the last chapter, we start by constructing a Routh table for $P(\lambda)$,

$$
\begin{array}{c|cc}
\lambda^{3} & 1 & b \\
\lambda^{2} & a & c \\
\lambda^{1} & d & 0 \\
\lambda^{0} & c & 0
\end{array}
$$

where

$$
d=\frac{a b-c}{a}
$$

By Theorem 2.4.1, a necessary and sufficient condition for all of the roots of (3.10) to lie in the left half plane is that all the entries of the first column of the Routh array are of the same sign and different from zero. This condition is equivalent to demanding that $a>0, c>0$, and $d>0$. If we demand both $a>0$ and $d>0$ we must have $(a b-c)>0$. Let us reformulate this new condition in terms of three constants $c_{1}=a, c_{2}=a b-c$, and $c_{3}=c$.

A necessary and sufficient condition for (3.10) to have all of its roots in the LHP 
is for all three of the following inequalities to be satisfied,

$$
\left\{\begin{array}{l}
c_{1}=12+\frac{k}{x_{0}}>0 \\
c_{2}=20 \frac{k^{2}}{x_{0}^{2}}+(261-10 R) \frac{k}{x_{0}}+(242-110 R)+11 k x_{0}+102 x_{0}^{2}>0 \\
c_{3}=1-R+3 x_{0}^{2}>0 .
\end{array}\right.
$$

We can now prove that the equilibrium point $x_{1}$ is locally stable for a subset of $G$ and that the equilibriums points $x_{2}$ and $x_{3}$ are unstable for all $(R, k) \in G$.

First, we consider the stability of the equilibrium point $x_{1}$.

Theorem 3.3.1. The equilibrium point $x_{1}$ is locally stable for all $(R, k) \in H$, where $H \subset G$ such that $H=\left\{(R, k) \mid k>P_{k}(R)\right\}$ and unstable for all $(R, k)$ in $G \backslash H$. The function $P_{k}(R)$ can be approximated by the second order polynomial $P_{k}(R) \approx$ $0.0009 R^{2}+0.2122 R-4.2175$.

Proof. In order for $x_{1}$ to be locally stable, it is both necessary and sufficient that $x_{1}$ satisfy all three inequalities given in (3.11). The first inequality, $c_{1}=12+\frac{k}{x_{1}}>0$, is easily satisfied since $x_{1}>0$ by (3.7). The third inequality, $c_{3}=3 x_{1}^{2}-(R-1)>0$, is also satisfied for all $(R, k) \in G$. Since $x_{1}>\sqrt{\frac{R-1}{3}}$ by $(3.7)$, we have

$$
c_{3}=3 x_{1}^{2}-(R-1)>3\left(\frac{R-1}{3}\right)-(R-1)=0,
$$

thus $c_{3}>0$ for all $(R, k) \in G$. The condition $c_{2}>0$ is equivalent to the following condition on a fourth-order polynomial $P_{2}$,

$$
P_{2}(R, k)=102 x_{1}^{4}+11 k x_{1}^{3}+(242-110 R) x_{1}^{2}+(261-10 R) k x_{1}+20 k^{2}>0
$$

where $x_{1}$ is given by

$$
x_{1}=2 \sqrt{\frac{R-1}{3}} \cos \left(\frac{\phi}{3}\right)
$$


and

$$
\cos (\phi)=\frac{-k}{2 \sqrt{\left(\frac{|R-1|}{3}\right)^{3}}} .
$$

The behavior of this function $P_{2}(R)$ can be seen in Figure 3.4. There is a clear region in $G$ where $P_{2}>0$, and a region where $P_{2}<0$, separated by a single curve along which $P_{2}=0$. Thus, the problem of finding the region in $G$ where $x_{1}$ is locally stable reduces to finding the line along which $P_{2}=0$. In Figure 3.5 we have located several such points where $P_{2}=0$ and fitted the data to a second-order polynomial of the form

$$
k=P_{k}(R) \approx 0.0009 R^{2}+0.2122 R-4.2175
$$

Thus, we can conclude that $x_{1}$ is locally stable for all points in $G$ which satisfy the inequality $k>P_{k}(R)$ where $P_{k}(R) \approx 0.0009 R^{2}+0.2122 R-4.2175$, and unstable elsewhere.

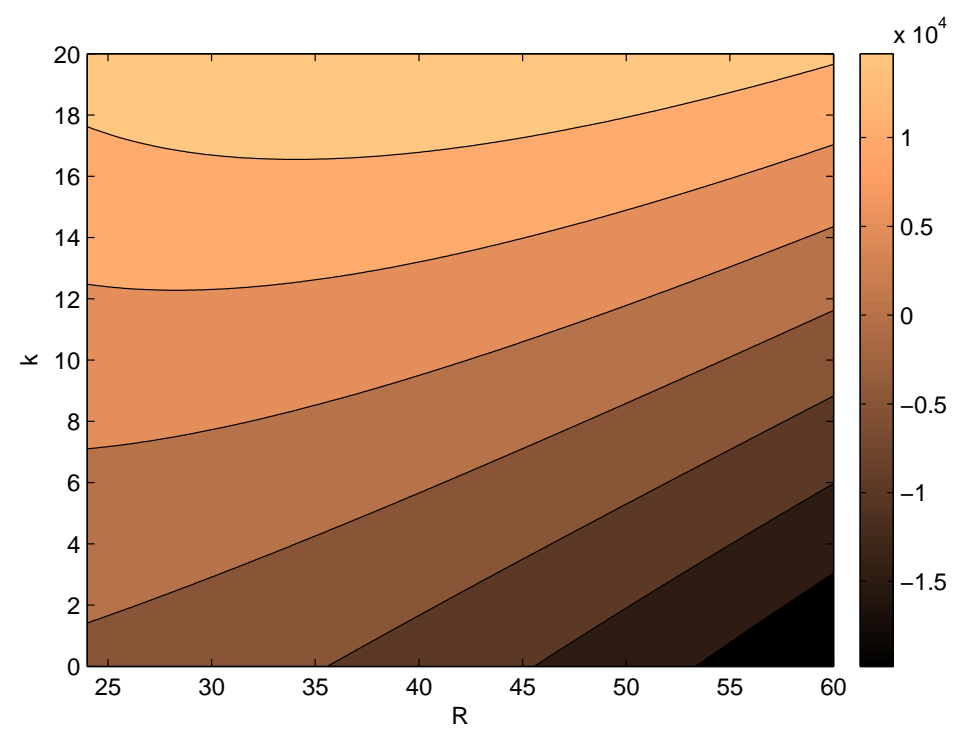

Figure 3.4: Behavior of $P_{2}(R, k)$ 


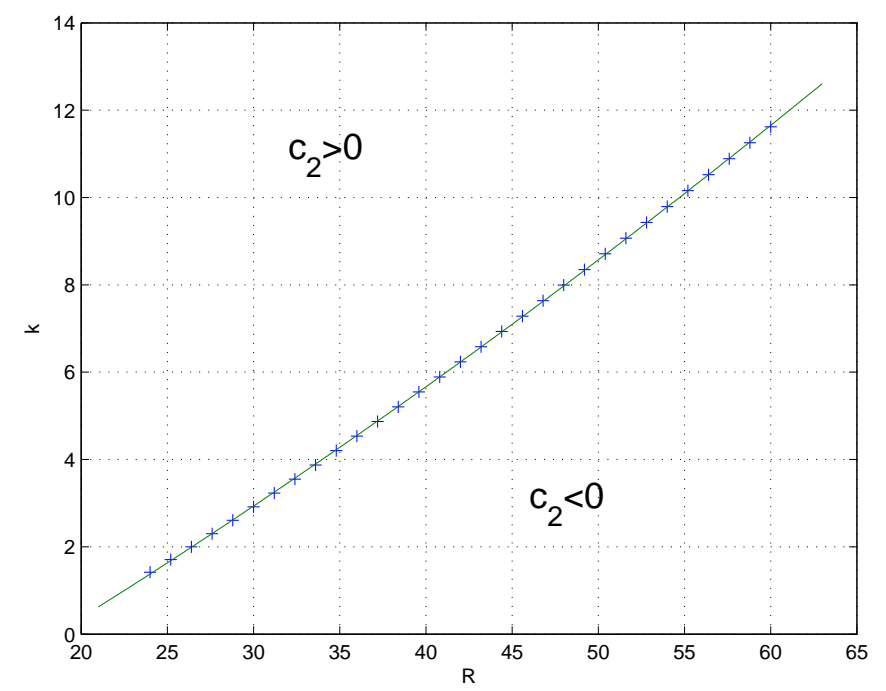

Figure 3.5: Regions of stability $\left(c_{2}>0\right)$ and instability $\left(c_{2}<0\right)$ for $x_{1}$

Next, we turn our attention to $x_{2}$ and $x_{3}$, which are both unstable.

Theorem 3.3.2. The equilibrium point $x_{2}$ is unstable for all $(R, k) \in G$.

Proof. Assume that $x_{2}$ is locally stable and derive a contradiction. By (3.8), for all $(R, k) \in G$ the point $x_{2}$ is located in the range

$$
-2 \sqrt{\frac{|R-1|}{3}}<x_{2}<-\sqrt{|R-1|} .
$$

If $x_{2}$ is locally stable, then by the first inequality of (3.11) we have $x_{2}>-\frac{k}{12}$. Thus $x_{2}$ must lie in the range

$$
-\frac{k}{12}<x_{2}<-\sqrt{R-1}
$$

which can be satisfied only if $k>12 \sqrt{R-1}$. However, since $12 \sqrt{R-1}$ is a monotonically increasing function of $R$ with lower bound $12 \sqrt{R_{\min }-1}=12 \sqrt{23}$ and $k_{\max }=20$, we see that $k<12 \sqrt{R-1}$ for all $(R, k) \in G$. We have arrived at a contradiction, so we conclude that $x_{2}$ is locally unstable for all $(R, k) \in G$. 
Theorem 3.3.3. The equilibrium point $x_{3}$ is unstable for all $(R, k) \in G$.

Proof. Assume that $x_{3}$ is locally stable and derive a contradiction. By (3.9), for all $(R, k) \in G$ the equilibrium point $x_{3}$ falls in the range

$$
0<x_{3}<\sqrt{\frac{R-1}{3}} .
$$

If $x_{3}$ is locally stable, then by the third inequality in (3.11) we have $1-R+3 x_{3}^{2}>0$, which implies

$$
-\sqrt{\frac{R-1}{3}}>x_{3} \text { or } x_{3}>\sqrt{\frac{R-1}{3}} .
$$

Since $x_{3}$ cannot satisfy the either inequality in the range $\left(0, \sqrt{\frac{R-1}{3}}\right)$, we have arrived at a contradiction and conclude that $x_{3}$ is locally unstable for all values of $(R, k) \in$ $G$

As such, we can conclude that $x_{1}$ is the only locally stable equilibrium point in any subset of $G$. In the next chapter, we illustrate the global stability of $x_{1}$ in a subset of $\mathbb{R}^{3}$. 


\section{CHAPTER 4}

\section{GLOBAL STABILITY}

In this chapter we evaluate the global stability of an equilibrium point using a geometric approach similar to that found in [14]. After presenting a few basic definitions and developing some necessary mathematical tools, we derive a sufficient condition on the feedback gain $k$ such that the equilibrium point is globally stable.

\subsection{Introduction}

Here we present an outline of a geometric approach to global-stability problems as discussed in [13], from which some of this section is adapted. Consider the differential equation

$$
\dot{x}=f(x),
$$

where $x \in \mathbb{R}^{n}$ and $f$ is a map from $D \subset \mathbb{R}^{n}$ to $\mathbb{R}^{n}$. The solution to (4.1) consists of a set of trajectories $x\left(t, x_{0}\right)$, where $x_{0} \in D$ is the initial value $x\left(0, x_{0}\right)=x_{0}$ and $t \in \mathbb{R}$. Every trajectory is uniquely determined by its initial value.

As discussed in previous chapters, all points $\bar{x} \in D$ which satisfy the equation $f(\bar{x})=0$ for all time $t$ are called equilibrium points in $D$. An equilibrium point is called locally stable if for each neighborhood $U$ of $\bar{x}$ there exists a neighborhood $V$ of $\bar{x}$ such that $x(t, V) \subset U$ for all $t>0$; it attracts points in a neighborhood $W$ if $x\left(t, x_{0}\right) \rightarrow \bar{x}$ as $t \rightarrow \infty$ for each $x_{0} \in K \subset D$ [13]. We may call $K$ an absorbing set. If $\bar{x}$ is locally stable and it attracts points in a neighborhood, we can call $\bar{x}$ asymptotically stable, and the union of all points which it attracts is called the basin of attraction. An equilibrium point is said to be globally stable with respect to an open set $D_{1}$ if it is asymptotically stable and its basin of attraction contains $D_{1}$ [13]. 
Local stability can often be established by linearizing the equation (4.1) about $\bar{x}$, provided that $f$ is continuous and differentiable. However, this linear approximation is only valid in a small neighborhood of $\bar{x}$, so local stability analysis only gives us insight into the behavior of the system near $\bar{x}$. We wish to place conditions on $f$ such that the equilibrium point $\bar{x}$ is globally stable with respect to an open set $D_{1}$. First, we need to develop some mathematical tools - specifically, compound matrices and the Lozinskii logarithmic norm.

Compound matrices are discussed extensively in [14] and [15], from which some of the following material is adapted. Let $A$ be any $n \times m$ matrix with real or complex entries $a_{i j}$, and let $x_{i_{1} \ldots i_{k}}^{j_{1} \ldots j_{k}}$ represent the minor of $A$ determined by the rows $\left(i_{1}, \ldots, i_{k}\right)$ and the columns $\left(j_{1}, \ldots, j_{k}\right)$, where $1 \leq i_{1}<i_{2}<\cdots<i_{k} \leq n$ and $1 \leq j_{1}<j_{2}<$ $\cdots<j_{k} \leq m$. We can then define the $k$-th multiplicative compound matrix $A^{(k)}$ of $A$ as the $\left(\begin{array}{l}n \\ k\end{array}\right) \times\left(\begin{array}{l}m \\ k\end{array}\right)$ matrix whose entries, written in lexicographic order, are $x_{i_{1} \ldots i_{k}}^{j_{1} \ldots j_{k}}$. For $n=m=3$ and $k=2$, we have

$$
A^{(2)}=\left(\begin{array}{rrr}
x_{1,2}^{1,2} & x_{1,2}^{1,3} & x_{1,2}^{2,3} \\
x_{1,3}^{1,2} & x_{1,3}^{1,3} & x_{1,3}^{2,3} \\
x_{2,3}^{1,2} & x_{2,3}^{1,3} & x_{2,3}^{2,3}
\end{array}\right) .
$$

The name multiplicative compound matrix comes from the property

$$
(A B)^{(k)}=A^{(k)} B^{(k)}
$$

for any matrices $A$ and $B$ which can be multiplied.

For $m=n$, the $k$-th additive compound matrix $A^{[k]}$ of $A$ is the $\left(\begin{array}{l}n \\ k\end{array}\right) \times\left(\begin{array}{l}n \\ k\end{array}\right)$ matrix defined by

$$
A^{[k]}=\left.D(I+h A)^{(k)}\right|_{h=0},
$$


where $D$ denotes differentiation with respect to $h$. In general, each entry of $A^{[k]}$ is a linear combination of the entries of $A$. For the case $n=3$ and $k=2$, which is the most important case for our work, the second additive compound matrix of $A=\left(a_{i j}\right)$ is

$$
A^{[2]}=\left(\begin{array}{ccc}
a_{11}+a_{22} & a_{23} & -a_{13} \\
a_{32} & a_{11}+a_{33} & a_{12} \\
-a_{31} & a_{21} & a_{22}+a_{33}
\end{array}\right) .
$$

The term additive compound comes from the property

$$
(A+B)^{[k]}=A^{[k]}+B^{[k]} .
$$

The names multiplicative compound matrix and additive compound matrix also correspond to the spectral properties of compound matrices. If $\sigma(A)=\left\{\lambda_{1}, \cdots, \lambda_{n}\right\}$ is the spectrum of $A$, then $\sigma\left(A^{(2)}\right)=\left\{\lambda_{i} \cdot \lambda_{j} \mid 1 \leq i<j \leq n\right\}$ and $\sigma\left(A^{[2]}\right)=\left\{\lambda_{i}+\lambda_{j} \mid 1 \leq i<\right.$ $j \leq n\}$ are the spectra of $A^{(2)}$ and $A^{[2]}$ respectively. For a more detailed discussion of general compound matrices, see reference [15].

The other mathematical tool that we will need is the Lozinskii logarithmic norm, which intuitively gives us a measure of how the distance between points changes under the action of a vector field. If $\|\cdot\|$ is any vector norm in $\mathbb{R}^{n}$ and also the matrix norm which it induces, the Lozinskii measure is then given by the right-handed derivative

$$
\mu(A)=\lim _{h \rightarrow 0^{+}} \frac{\|I+h A\|-1}{h} .
$$

The value of $\mu(A)$ depends on the norm that is chosen. Under the infinity norm $\|x\|=\sup _{i}\left|x_{i}\right|$, the Lozinskii norm $\mu_{\infty}$ of $A=\left(a_{i j}\right)$ is

$$
\mu_{\infty}(A)=\sup _{i}\left[\operatorname{Re}\left(a_{i i}\right)+\sum_{j \neq i}\left|a_{i j}\right|\right] .
$$


Under the $L^{1}$-norm $\|x\|=\Sigma_{i}\left|x_{i}\right|$, the Lozinskii measure $\mu_{1}$ is

$$
\mu_{1}(A)=\sup _{j}\left[\operatorname{Re}\left(a_{j j}\right)+\sum_{i \neq j}\left|a_{i j}\right|\right] .
$$

If the Euclidean (or $L^{2}$ ) norm $\|x\|=\left(\Sigma_{i}\left|x_{i}\right|^{2}\right)^{1 / 2}$ is used, the Lozinskii norm is given by the largest eigenvalue of $\left(A^{*}+A\right) / 2$. Finally, if we restrict our attention to $\mathbb{R}^{3}$ with vectors $(u, v, w)$ and use the norm $\|(u, v, w)\|=\max \{|u|,|v|+|w|\}$ introduced in [14], then the Lozinskii norm $\mu(A)$ has an upper bound

$$
\mu(A) \leq \sup \left\{g_{1}, g_{2}\right\}
$$

where $g_{1}$ and $g_{2}$ are given by

$$
\begin{aligned}
& g_{1}=\mu_{1}\left(A_{11}\right)+\left\|A_{12}\right\| \\
& g_{2}=\left\|A_{21}\right\|+\mu_{1}\left(A_{22}\right) .
\end{aligned}
$$

Note that $\left\|A_{12}\right\|$ and $\left\|A_{21}\right\|$ are taken with respect to the $L^{1}$-norm and we have written the $3 \times 3$ matrix $A$ in block form,

$$
A=\left(\begin{array}{ll}
A_{11} & A_{12} \\
A_{21} & A_{22},
\end{array}\right) .
$$

where $A_{22}$ is a $2 \times 2$ matrix. We can now use compound matrices and the Lozinskii norm in two autonomous convergence theorems.

\subsection{Autonomous Convergence Theorems}

Consider a set $D \subset \mathbb{R}^{3}$ which contains an equilibrium point $\bar{x}$ of (4.1). If each closed curve in $D$ can be continuously deformed into a point in $D$, then we call $D$ simply connected. The global stability problem can be stated as follows: assume that 
- $\left(C_{1}\right) D$ is simply connected;

- $\left(C_{2}\right)$ there is a compact absorbing set $K \subset D$;

- $\left(C_{3}\right) \bar{x}$ is the only equilibrium of $(4.1)$ in $D$.

We need conditions under which the local stability of $\bar{x}$ implies global stability with respect to $D$.

This problem is often addressed by the construction of a Lyapunov function, which serves somewhat the same role as the Hamiltonian in classical mechanics. However, Lyapunov functions are typically difficult to construct and often require a great deal of physical intuition about the behavior of the system. As of yet there are no general methods for constructing Lyapunov functions of broad classes of systems, so each system must be treated almost independently.

When no Lyapunov function is available, we may use one of the so-called autonomous convergence theorems to prove global stability. Such theorems are constructed around a Bendixon criteria, which is a condition that rules out the existence of periodic orbits in $D$ when satisfied. For $n=2$ and $D=\mathbb{R}^{2}$, the classical Bendixon result states that if $\operatorname{div}(f)<0$ in $\mathbb{R}^{2}$, then (4.1) has no nonconstant periodic solutions.

For $n>2$, there are several higher-dimensional Bendixon conditions that can be used to rule out the existence of periodic orbits in a set $D$. In [13], one such Bendixon criteria is established, which we present here without proof. Let $x \rightarrow P(x)$ be an $\left(\begin{array}{l}n \\ 2\end{array}\right) \times\left(\begin{array}{l}n \\ 2\end{array}\right)$ matrix-valued function that is continuous and once-differentiable for all $x \in D$, and assume that $P^{-1}(x)$ exists and is continuous for all $x \in K$, where $K$ is the compact absorbing set which is assumed to exist under $\left(C_{2}\right)$ [14]. We can then 
define $\bar{q}_{2}$ by

$$
\bar{q}_{2}=\varlimsup_{t \rightarrow \infty} \sup _{x_{0} \in K} \frac{1}{t} \int_{0}^{t} \mu\left\{B\left[x\left(\tau, x_{0}\right)\right]\right\} d \tau
$$

where

$$
B=P_{f} P^{-1}+P J^{[2]} P^{-1}
$$

Note that $P_{f}$ is obtained by replacing each entry of $P$ by its derivative in the direction of the vector field $f, J^{[2]}$ is the second additive compound matrix of the Jacobian $J$, and the Lozinskii measure $\mu(B)$ is taken with respect to a vector norm $\|\cdot\|$ in $\mathbb{R}^{N}$, with $N=\left(\begin{array}{l}n \\ 2\end{array}\right)$. We can now state a theorem regarding the global stability of the equilibrium point $\bar{x}$.

Theorem 4.2.1. Under assumptions $\left(C_{1}\right),\left(C_{2}\right)$, and $\left(C_{3}\right)$ if

$$
\bar{q}_{2}<0
$$

then the unique equilibrium point $\bar{x}$ in $D$ is globally stable, provided it is locally stable.

Alternatively, we can use a theorem from [15] to help in our demonstration of global stability. If we take $P$ to be the identity matrix, then $B=J^{[2]}$ and we can put a condition on the sign of the Lozinskii norm for global stability.

Theorem 4.2.2. Suppose that one of the inequalities

$$
\mu\left(J^{[2]}\right)<0, \mu\left(-J^{[2]}\right)<0
$$

holds for all $x$ on steady-state trajectories of (4.1). Then (4.1) has no nonconstant periodic solutions.

We can now apply these theorems to the controlled Lorenz system in order to demonstrate global stability. 


\subsection{Global Stability Analysis}

In this section we present a theorem regarding the global stability of the equilibrium point $\bar{x}=x_{1}$ of $(3.1)$, which is the only locally stable fixed point for any $(R, k) \in G$.

Consider a set $D \subset \mathbb{R}^{3}$ that lies in the first octant such that $\beta>x, y, z>\alpha>0$ and $\bar{x}=x_{1} \in D$. Our choice of $D$ eliminates the possibility of trajectories which intersect the $z$-axis, since any trajectory which intersects the $z$-axis quickly evolves to the origin. We assume that $\left(C_{1}\right) D$ is simply connected, $\left(C_{2}\right)$ there exists a compact absorbing set $K \subset D$, and $\left(C_{3}\right) \bar{x}$ is the only equilibrium point in $D$. Recall that we only consider values of $(R, k)$ in the parameter space $G=\left\{(R, k) \mid R_{\text {min }} \leq R \leq\right.$ $\left.R_{\max }, k_{\min } \leq k \leq k_{\max }\right\}$

Theorem 4.3.1. Under assumptions $\left(C_{1}\right),\left(C_{2}\right)$, and $\left(C_{3}\right)$, there exists a subset of the parameter space $G$ for which the equilibrium point $\bar{x}=x_{1}$ is globally asymptotically stable in $D$.

Proof. The controlled Lorenz system is given by

$$
\left\{\begin{aligned}
\dot{x} & =10(y-x) \\
\dot{y} & =\left(R-k \frac{y}{z}\right) x-y-x z \\
\dot{z} & =x y-z
\end{aligned}\right.
$$

where $k$ is the feedback gain. The Jacobian $J=\frac{\partial f}{\partial x}$ of (4.12) is

$$
J(x, y, z)=\left(\begin{array}{ccc}
-10 & 10 & 0 \\
R-k \frac{y}{z}-z & -k \frac{x}{z}-1 & k \frac{x y}{z^{2}}-x \\
y & x & -1
\end{array}\right)
$$


The second additive compound $J^{[2]}$ of the Jacobian $J$ is

$$
J^{[2]}=\left(\begin{array}{ccc}
-k \frac{x}{z}-11 & k \frac{x y}{z^{2}}-x & 0 \\
x & -11 & 10 \\
-y & R-k \frac{y}{z}-z & -k \frac{x}{z}-2
\end{array}\right) .
$$

Let $P$ be a function $P(y, z)=\operatorname{diag}\left(1, \frac{y}{z}, \frac{y}{z}\right)$, such that $P^{-1}=\operatorname{diag}\left(1, \frac{z}{y}, \frac{z}{y}\right)$ and

$$
P_{f}=\operatorname{diag}\left(0,\left(\frac{y}{z}\right)_{f},\left(\frac{y}{z}\right)_{f}\right)=\left(0, \frac{\dot{y}}{z}-\frac{y \dot{z}}{z^{2}}, \frac{\dot{y}}{z}-\frac{y \dot{z}}{z^{2}}\right)
$$

The product $P_{f} P^{-1}$ is

$$
P_{f} P^{-1}=\left(0, \frac{\dot{y}}{y}-\frac{\dot{z}}{z}, \frac{\dot{y}}{y}-\frac{\dot{z}}{z}\right) .
$$

Under this choice for $P$, the matrix $B=P_{f} P^{-1}+P J^{[2]} P^{-1}$ becomes

$$
B=\left(\begin{array}{ccc}
-k \frac{x}{z}-11 & k \frac{x}{z}-\frac{x z}{y} & 0 \\
\frac{x y}{z} & -11+\frac{\dot{y}}{y}-\frac{\dot{z}}{z} & 10 \\
-\frac{y^{2}}{z} & R-k \frac{y}{z}-z & -k \frac{x}{z}-2+\frac{\dot{y}}{y}-\frac{\dot{z}}{z},
\end{array}\right)
$$

We have the block matrices $B_{11}=-k \frac{x}{z}-11, B_{12}=\left(k \frac{x}{z}-\frac{x z}{y}, 0\right), B_{21}^{T}=\left(\frac{x y}{z},-\frac{y^{2}}{z}\right)$, and

$$
B_{22}=\left(\begin{array}{cc}
-11+\frac{\dot{y}}{y}-\frac{\dot{z}}{z} & 10 \\
R-k \frac{y}{z}-z & -k \frac{x}{z}-2+\frac{\dot{y}}{y}-\frac{\dot{z}}{z} .
\end{array}\right) .
$$

If we denote the Lozinskii norm of $B$ under the vector norm $\|(u, v, w)\|=\max \{|u|,|v|+$ $|w|\}$ by $\mu(B)$ and the Lozinskii norm of $B$ under the $L^{1}$-norm by $\mu_{1}(B)$, then we have

$$
\mu(B) \leq \sup \left\{g_{1}, g_{2}\right\}
$$

where $g_{1}$ and $g_{2}$ are given by

$$
\begin{aligned}
& g_{1}=\mu_{1}\left(B_{11}\right)+\left\|B_{12}\right\|_{1} \\
& g_{2}=\left\|B_{21}\right\|_{1}+\mu_{1}\left(B_{22}\right) .
\end{aligned}
$$


For the controlled Lorenz system (4.12),

$$
\begin{aligned}
\mu_{1}\left(B_{11}\right) & =-k \frac{x}{z}-11 \\
\left\|B_{12}\right\|_{1} & =\left|k \frac{x}{z}-\frac{x y}{z}\right| \\
\left\|B_{21}\right\|_{1} & =\left|\frac{x y}{z}\right|+\left|\frac{y^{2}}{z}\right| \\
\mu_{1}\left(B_{22}\right) & =\frac{\dot{y}}{y}-\frac{\dot{z}}{z}+\max \left(-11+\left|R-k \frac{y}{z}-z\right|,-k \frac{x}{z}+8\right),
\end{aligned}
$$

such that $g_{1}$ and $g_{2}$ are

$$
\begin{aligned}
& g_{1}=-k \frac{x}{z}-11+\left|k \frac{x}{z}-\frac{x y}{z}\right| \\
& g_{2}=1+\frac{y^{2}}{z}+\frac{\dot{y}}{y}+\max \left(\theta_{1}, \theta_{2}\right),
\end{aligned}
$$

where $\theta_{1}=-11+\left|R-k \frac{y}{z}-z\right|, \theta_{2}=-k \frac{x}{z}+8$, and we have used (4.12)

$$
\frac{\dot{z}}{z}=\frac{x y}{z}-1
$$

to simplify $g_{2}$.

In order to put an upper bound on $\bar{q}_{2}$, we need to put an upper bound on $g_{1}$ and $g_{2}$. It is convenient to divide $\mu$ into a continuous piecewise function such that $\mu_{T}$ is the Lozinskii norm evaluated during the system's transient response (for $t \leq T$ ) and $\mu_{s s}$ is the Lozinskii norm measured after the system has settled into a steady-state trajectory (for $t>T$ ),

$$
\mu(B)= \begin{cases}\mu_{T}(B) & \text { for } t \leq T \\ \mu_{s s}(B) & \text { for } t>T\end{cases}
$$

where $\mu_{T}=\mu_{s s}$ at $t=T$. From (4.8) we have

$$
\bar{q}_{2}=\varlimsup_{t \rightarrow \infty} \sup _{x_{0} \in K} \frac{1}{t} \int_{0}^{t} \mu(B) d \tau=\varlimsup_{t \rightarrow \infty} \sup _{x_{0} \in K}\left[\frac{1}{t} \int_{0}^{T} \mu_{T}(B) d \tau+\frac{1}{t} \int_{T}^{t} \mu_{s s}(B) d \tau\right]
$$


Since $x, y$, and $z$ and their time derivatives are bounded, then $g_{1}$ and $g_{2}$ are bounded above and $\mu_{T}(B)$ is bounded above by a constant $M_{T}$. As such,

$$
\bar{q}_{2} \leq \varlimsup_{t \rightarrow \infty} \sup _{x_{0} \in K}\left[\frac{1}{t} \int_{0}^{T} M_{T} d \tau+\frac{1}{t} \int_{T}^{t} \mu_{s s}(B) d \tau\right]=\varlimsup_{t \rightarrow \infty} \sup _{x_{0} \in K} \frac{1}{t} \int_{T}^{t} \mu_{s s}(B) d \tau
$$

since the first term goes to zero as $t \rightarrow \infty$.

From (4.13) we have $\mu_{s s}(B) \leq \sup \left\{g_{1}, g_{2}\right\}$. We will consider three separate cases for the upper bound on $\mu_{s s}$. Namely, we consider $\left(g_{1}>g_{2}\right),\left(g_{2}>g_{1}\right) \wedge\left(\theta_{1}>\theta_{2}\right)$, and finally $\left(g_{2}>g_{1}\right) \wedge\left(\theta_{2}>\theta_{1}\right)$, where $\theta_{1}$ and $\theta_{2}$ are the first and second arguments of the max function in $g_{2}$, respectively. We only want to consider values of $(R, k) \in G$ for which the equilibrium point $\bar{x}$ is locally stable, which from Theorem 3.3.1 is the subset $H \subset G$ where $H=\left\{(R, k) \mid k>P_{k}(R)\right\}$.

- Case $1\left(g_{1}>g_{2}\right)$ : Let $(R, k) \in H \cap H_{1}$, where $H_{1}=\left\{(R, k) \mid k \geq \beta_{s s}>y\right\}$ and $\beta_{s s}$ is the largest value that $y$ can take in the steady-state. Then we have $k \frac{x}{z}>k \frac{x y}{z}$, such that

$$
g_{1}=-k \frac{x}{z}-11+\left|k \frac{x}{z}-\frac{x y}{z}\right|=-11-\frac{x y}{z} .
$$

Since $x, y, z>\alpha>0$ in $D, g_{1}<M_{1}<0$ for all $x, y, z \in D$, where $M_{1}$ is a constant. So $\mu_{s s}(B) \leq \sup \left\{g_{1}, g_{2}\right\}=g_{1}<M_{1}$, which implies $\mu_{s s}(B)<M_{1}$.

Thus (4.18) becomes

$$
\bar{q}_{2} \leq \varlimsup_{t \rightarrow \infty} \sup _{x_{0} \in K} \frac{1}{t} \int_{T}^{t} \mu_{s s}(B) d \tau<\varlimsup_{t \rightarrow \infty} \sup _{x_{0} \in K} \frac{1}{t} \int_{T}^{t} M_{1} d \tau=M_{1}<0 .
$$

Since $\bar{x}$ is locally stable for all $(R, k) \in H \cap H_{1}$ and $\bar{q}_{2}<0, \bar{x}$ is globally stable in $D$ under assumptions $\left(C_{1}\right),\left(C_{2}\right)$, and $\left(C_{3}\right)$ by Theorem 4.2.1.

- Case $2\left(g_{2}>g_{1}\right.$ and $\left.\theta_{1}>\theta_{2}\right)$ : Let $(R, k) \in H$. Since we are considering a steadystate solution, we have $x \approx y$ and $z \approx x^{2}$, such that we can set $\frac{y^{2}}{z}=1+\delta_{1}$ 
where $\delta_{1}$ is small. Furthermore, in the steady-state we have the expression

$$
\left|R-k \frac{y}{z}-z\right| \approx\left|R-k \frac{1}{x}-x^{2}\right|=\left|\frac{1}{x}\left(R x-k-x^{3}\right)\right| \approx\left|\frac{1}{x}(x)\right|=1,
$$

where we have used (3.4) in the approximation. Thus we can set $\left|R-k \frac{y}{z}-z\right|=$ $1+\delta_{2}$, where $\delta_{2}$ is small. Now we have

$$
g_{2}=-8+\delta_{1}+\delta_{2}+\frac{\dot{y}}{y}
$$

which by $\mu_{s s}(B) \leq \sup \left\{g_{1}, g_{2}\right\}=g_{2}$ implies

$$
\bar{q}_{2} \leq \varlimsup_{t \rightarrow \infty} \sup _{x_{0} \in K} \frac{1}{t} \int_{T}^{t} g_{2} d \tau=-8+\delta_{1}+\delta_{2}+\varlimsup_{t \rightarrow \infty} \frac{1}{t} \frac{\log [y(t)]}{\log [y(T)]} .
$$

Since $y(t)$ is bounded, $\log [y(t)]$ is bounded and the last term vanishes as $t \rightarrow \infty$. As such, $\bar{q}_{2} \leq-8+\left(\delta_{1}+\delta_{2}\right)<0$ which holds for small $\delta_{1}$, $\delta_{2}$. Since $\bar{q}_{2}<0$ and $\bar{x}$ is locally stable for all $(R, k) \in H, \bar{x}$ is globally stable in $D$ under assumptions $\left(C_{1}\right),\left(C_{2}\right)$, and $\left(C_{3}\right)$ by Theorem 4.2.1.

- Case $3\left(g_{2}>g_{2}\right.$ and $\left.\theta_{2}>\theta_{1}\right)$ : Let $(R, k) \in H \cap H_{2}$, where $H_{2}=\{(R, k) \mid k>$ $\left.(10+\Delta) \frac{\gamma_{s s}}{\alpha_{s s}}>10 \frac{z}{x}\right\}$, where $\alpha_{s s}>0$ is the largest value that $x$ can take in the steady-state, $\gamma_{s s}>0$ is the largest value that $z$ can take in the steady-state, and $\Delta>0$ is a constant. Since we are considering the system in steady-state, we can again assume $x \approx y$ such that $\frac{y^{2}}{z}=1+\delta_{3}$, where $\delta_{3}$ is small. Then $g_{2}$ becomes

$$
g_{2}=9+\frac{y^{2}}{z}-k \frac{x}{z}+\frac{\dot{y}}{y} \leq 10+\delta_{3}-k \frac{\alpha_{s s}}{\gamma_{s s}}+\frac{\dot{y}}{y}
$$

such that

$$
\bar{q}_{2} \leq \varlimsup_{t \rightarrow \infty} \sup _{x_{0} \in K} \frac{1}{t} \int_{T}^{t} g_{2} d \tau \leq 10+\delta_{3}-k \frac{\alpha_{s s}}{\gamma_{s s}}+\varlimsup_{t \rightarrow \infty} \frac{1}{t} \frac{\log [y(t)]}{\log [y(T)]}<\delta_{3}-\Delta<0,
$$

which holds if we select $\Delta>\delta_{3}$. Since $\bar{q}_{2}<0$ and $\bar{x}$ is locally stable for all $(R, k) \in H \cap H_{2}, \bar{x}$ is globally stable in $D$ under assumptions $\left(C_{1}\right),\left(C_{2}\right)$, and $\left(C_{3}\right)$ by Theorem 4.2.1. 
Thus, there exists a subset of the parameter space $H$ in which the equilibrium point $\bar{x}=x_{1}$ is globally stable in $D$.

\subsection{Numerical Results}

Alternatively, we may use Theorem 4.2.2 to rule out the existence of periodic orbits in the state space. Shown in Figures 4.1 and 4.2 are modified Feigenbaum diagrams which illustrate the behavior of the Lozinskii norm $\mu\left(J^{[2]}\right)$ in the $L^{2}$-norm for different values of $R$ and $k$. For each plot, a value was chosen for $k$ and $R$ was allowed to vary; the system was allowed to evolve for $t_{f}=30$. The darker the color, the more often the system visited that range of values in its evolution. The transient response of the system is shown in lighter colors.

In Figure 4.1, in which the feedback gain is set to $k=20$, we see that the value of $\mu\left(J^{[2]}\right)$ is indeed negative for a large range in $R$ along each trajectory after a transient response. By Theorem 4.2.2, if $\mu\left(J^{[2]}\right)<0$ for the steady-state response of the system, then no periodic orbits exist in the state space, and any trajectories which become trapped in the absorbing set $K$ will evolve towards the unique fixed point in $D$. As such, a feedback gain of $k=20$ is strong enough to push the system into global stability for all the ranges of $R$ considered. However, in Figure 4.2 we see that a controller with feedback gain $k=10$ is only capable of establishing global stability for the equilibrium point $\bar{x}$ for a smaller range of $R$ values. 


\subsection{Conclusions and Future Work}

In this chapter we established a rather conservative bound on the value of $k$ needed to bring about global stability. Our bound was a result of our choice of matrix $P$ and norm $\|\cdot\|$. It may be possible to select a different matrix $P$ and norm $\|\cdot\|$ that would give a tighter bound on the value of $k$ needed to push all trajectories in the state space towards the only locally stable equilibrium point. It may also be possible to construct a Lyapunov function for the controlled system, which would provide an alternative means of establishing global stability. The feasibility of implementing the quotient controller $u=-k \frac{y}{z}$ in an experimental setting is also yet to be determined. 


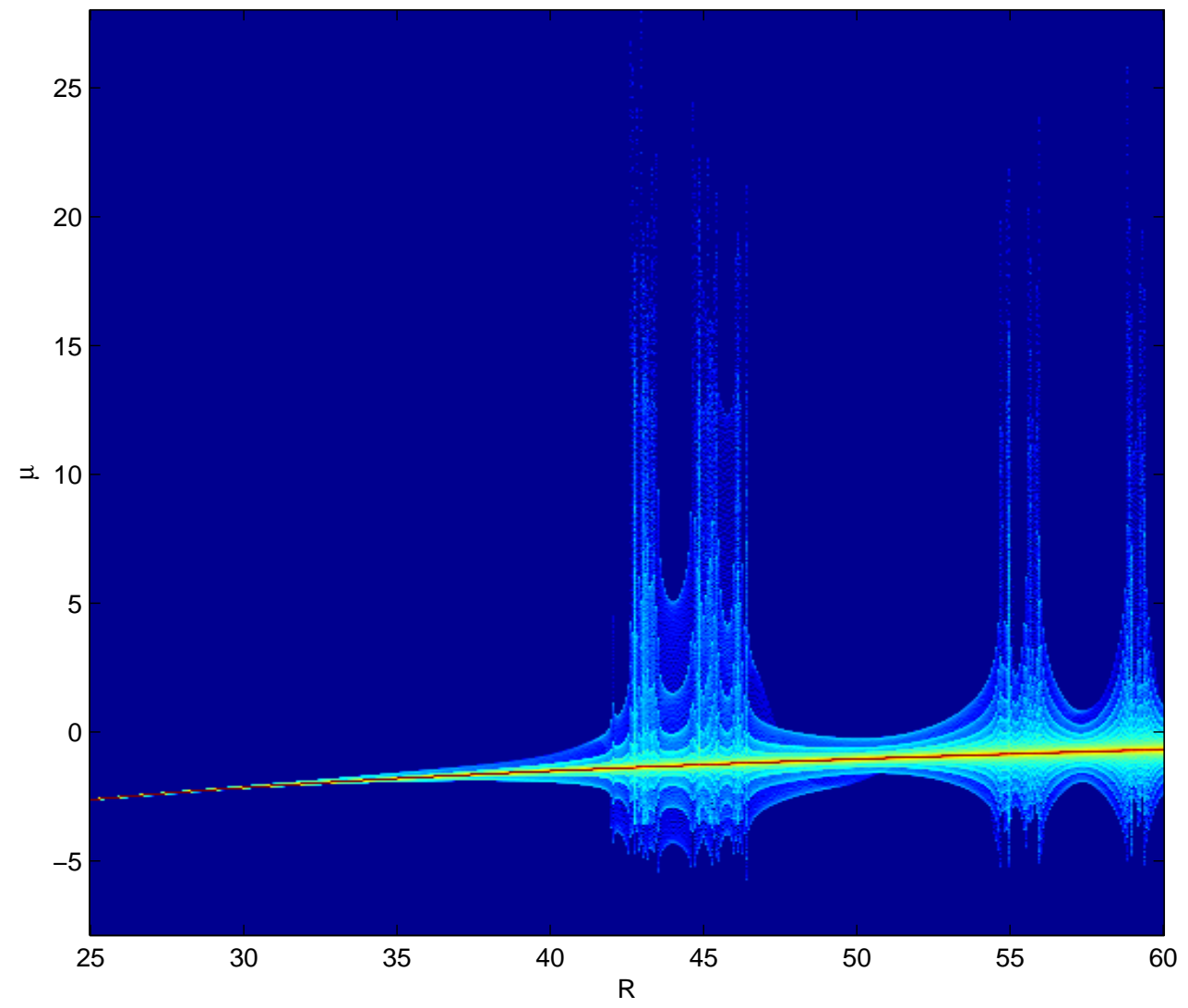

Figure 4.1: Lozinskii norm, $k=20, t_{f}=30$ 


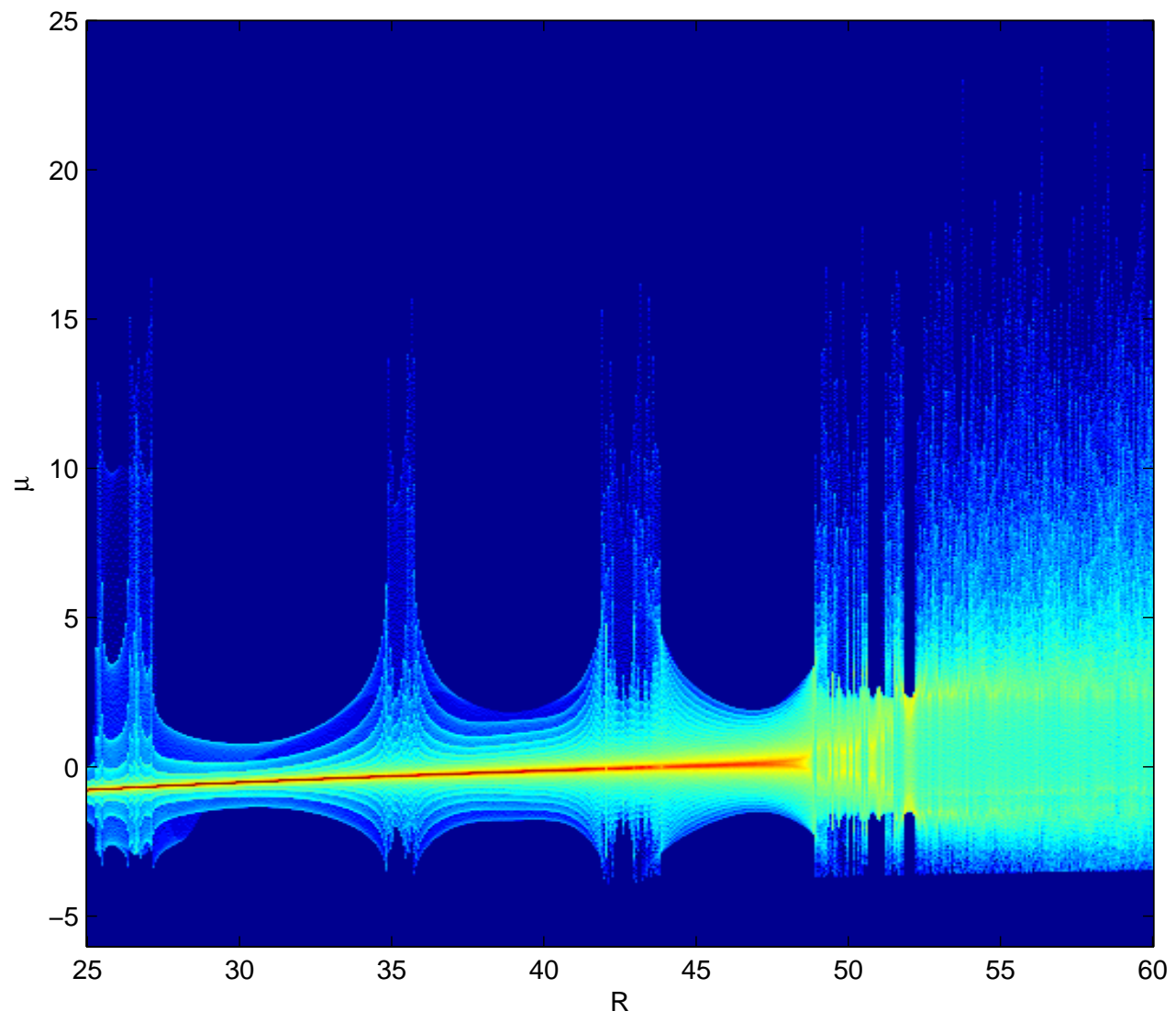

Figure 4.2: Lozinskii norm, $k=10, t_{f}=30$ 


\section{BIBLIOGRAPHY}

[1] W. Benenson, J.W. Harris, H. Stocker, H. Lutz, Handbook of Physics, Springer Publishing, New York, 2002.

[2] P. Berge, Y. Pomeau, and C. Vidal, Order within Chaos, Wiley-Interscience Publ., France, 1984.

[3] K.M. Cuomo, A.V. Oppenheim, and S.H. Strogatz, Synchronization of LorenzBased Chaotic Circuits with Applications to Communications, IEEE Transactions on Circuits and Systems - II: Analog and Digital Signal Processing 40 (1993), 626-633.

[4] W. L. Ditto, S. N. Rauseo, and M. L. Spano, Experimental Control of Chaos, Phys. Rev. Lett. 65 (1990), 3211 - 3214.

[5] P. Ehrhad, Ch. Karcher, and U. Muller, Dynamical Behavior of Natural Convection in a Double-Loop System, Experimental Heat Transfer 2 (1989), 13-26.

[6] F.R. Gantmacher, The Theory of Matrices, Chelsea Publishing Company, New York, 1959.

[7] H.Haken, Analogy between higher Instabilities in Fluids and Lasers, Phys. Lett. 53A (1975), 77-78.

[8] R. Hilborn, Chaos and Nonlinear Dynamics: An Introduction for Scientists and Engineers, Oxford University Press, USA, 1994.

[9] J.W. Harris, H. Stocker, Handbook of Mathematics and Computational Science, Springer Publishing, New York, 1998.

[10] D.W. Jordan and P. Smith, Nonlinear Ordinary Differential Equations, Oxford University Press, UK, 1977.

[11] E. Knobloch, Chaos in the Segmented Disk Dynamo, Physics Letters A 82 (1981), 439-440. 
[12] E.N. Lorenz, Deterministic Nonperiodic Flow, Journal of the Atmospheric Sciences 20 (1963), 130-141.

[13] M.Y. Li and J.S. Muldowney, A Geometric Approach to Global-Stability Problems, SIAM J. Math. Anal. 27 (1996), 1070-1083.

[14] M.Y. Li and L. Wang, Global Stability in Some SEIR Epidemic Models, Mathematical Approaches for Emerging and Reemerging Infectious Diseases, SpringerVerlag, New York, 2002.

[15] J.S. Muldowney, Compound Matrices and Ordinary Differential Equations, Rocky Mountain Journal of Mathematics 20 (1990), 857-872.

[16] E. Ott, C. Grebogi and Y.A. Yorke, Controlling Chaos, Phys. Rev. Lett. 64 (1990), 1196-1199.

[17] L. Prandtl, Essentials of Fluid Dynamics, Hafner Publishing Company, New York, 1952.

[18] K. Pyragas, Continuous control of chaos by self-controlling feedback, Phys. Lett. A 170 (1992), 421-428.

[19] J. Singer, Y-Z. Wang, and Haim H. Bau, Controlling a Chaotic System, Physical Review Letters 66 (1991), 1123-1125.

[20] R.T. Stefani, B. Shahian, C. J. Savant, and G. H. Hostetter, Design of Feedback Control Systems, Oxford University Press, New York, 2002.

[21] S.H. Strogatz, Nonlinear Dynamics and Chaos, Westview Press, New York, 2001.

[22] E. Suli and D. Mayers, An Introduction to Numerical Analysis, Cambridge University Press, UK, 2006.

[23] J. Tanner, State-feedback Control of a Single-loop Thermosyphon System via a Quotient Controller, Unpublished master's thesis, Georgia Southern University, Statesboro, GA, 2007.

[24] S. Whitaker, Introduction to Fluid Mechanics, Prentice-Hall, Inc., 1968. 
[25] S.W. Yuan, Foundations of Fluid Mechanics, Prentice-Hall, Inc., 1967. 


\section{APPENDIX A \\ FOURTH-ORDER RUNGE-KUTTA METHOD}

We present a discussion of the classical fourth-order Runge-Kutta method adapted from [22]. Consider the first-order differential equation of the form

$$
y^{\prime}=f(t, y)
$$

where $y=y(t)$ is a real-valued function of $t \in \mathbb{R}$ and $y^{\prime}=\frac{d y}{d t}$. The solution to (4.20) consists of a set of curves. If we combine equation (4.20) with an initial condition of the form

$$
y\left(x_{0}\right)=y_{0}
$$

then, if certain conditions (stated in Picard's theorem) regarding the growth of $f$ with respect to $y$ are satisfied, the initial value problem (4.20), (4.21) has a unique solution. For more detail see reference [22].

Suppose that the conditions of Picard's theorem are satisfied. In order to construct an approximate solution to the initial value problem we proceed step-by-step, using function values from one step to construct the next. Suppose that the integration is to be carried out on the interval $\left[t_{0}, t_{f}\right]$. The step size or mesh size $h$ is given by

$$
h=\frac{t_{f}-t_{0}}{N},
$$

where $N$ is a positive integer. The interval is divided into mesh points given by $t_{n}=t_{0}+n h$ where $n=0,1, \cdots, N$. For each $n$ we want a numerical approximation $y_{n}$ for $y\left(x_{n}\right)$, the exact solution at the mesh point $x_{n}$. 
The simplest way to construct a numerical approximation for $y\left(x_{n}\right)$ is known as Euler's method, defined by the iteration

$$
y_{n+1}=y_{n}+h f\left(x_{n}, y_{n}\right)
$$

where $n=0,1, \cdots, N-1$ and $y_{0}=y\left(x_{0}\right)$ is given as part of the initial value problem. This scheme is simple and cheap to implement in that it only requires one function evaluation per mesh point. However, it is only accurate to first order. We can achieve higher accuracy at the expense of efficiency by evaluating the function $f$ at several points between each pair of adjacent mesh points.

The standard workhorse method used to construct approximate solutions to initial value problems is the classical fourth-order Runge-Kutta method, defined by the iteration

$$
y_{n+1}=y_{n}+\frac{1}{6} h\left(k_{1}+2 k_{2}+2 k_{3}+k_{4}\right)
$$

where

$$
\left\{\begin{array}{l}
k_{1}=f\left(x_{n}, y_{n}\right) \\
k_{2}=f\left(x_{n}+\frac{1}{2} h, y_{n}+\frac{1}{2} h k_{1}\right) \\
k_{3}=f\left(x_{n}+\frac{1}{2} h, y_{n}+\frac{1}{2} h k_{2}\right) \\
k_{4}=f\left(x_{n}+h, y_{n}+h k_{3}\right) .
\end{array}\right.
$$

Here $k_{2}$ and $k_{3}$ represent approximations to the derivative $y^{\prime}$ at points on the solution curve between $\left(x_{n}, y\left(x_{n}\right)\right)$ and $\left(x_{n+1}, y\left(x_{n+1}\right)\right)$ and the second term in (4.22) is a weighted average. The total accumulated error is on the order of $h^{4}$. 


\section{APPENDIX B}

\section{MATLAB CODE}

Below are the M-files used to initialize, run, and plot the results of the Lorenz model, along with the code used to implement the fourth-order Runge-Kutta method.

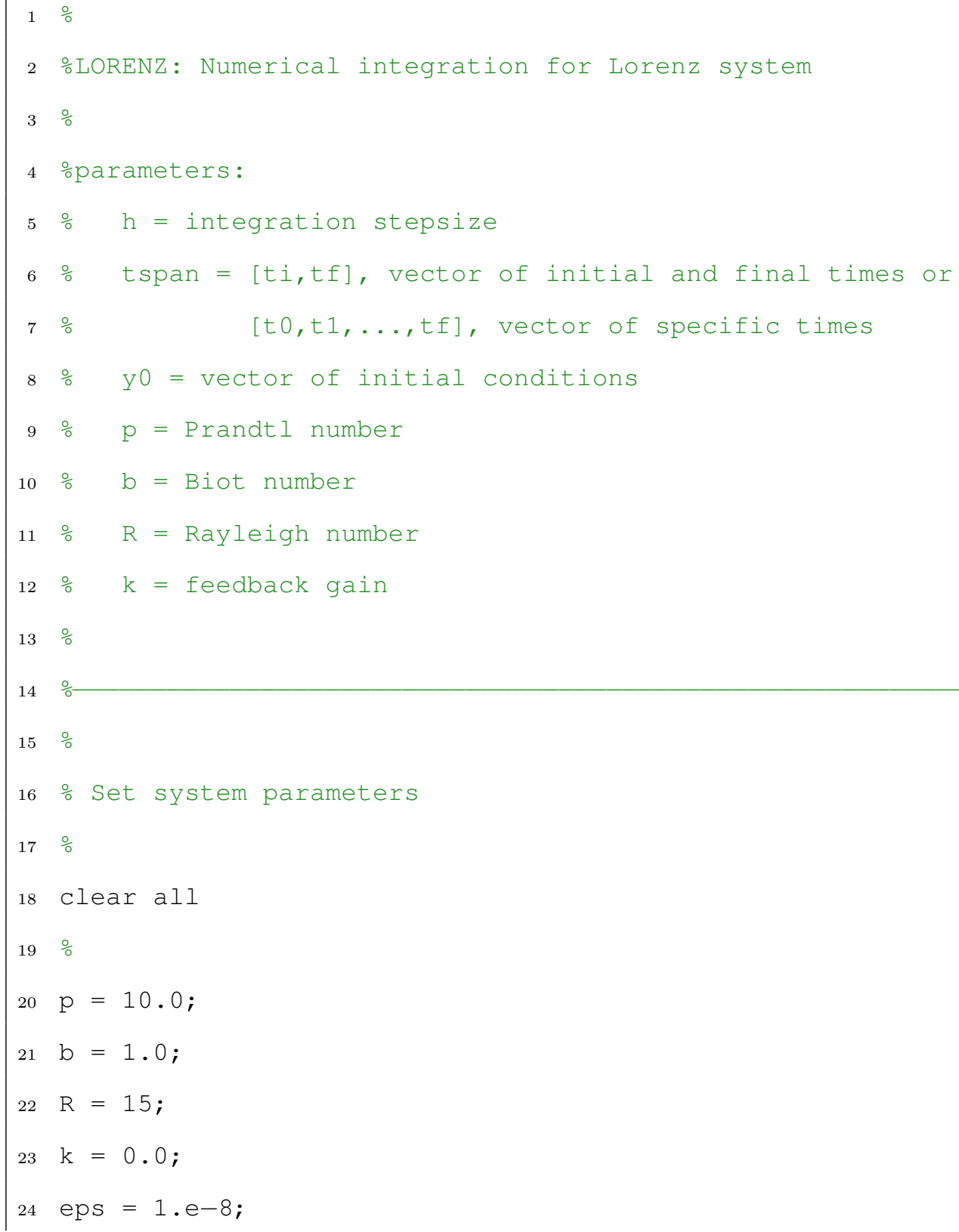




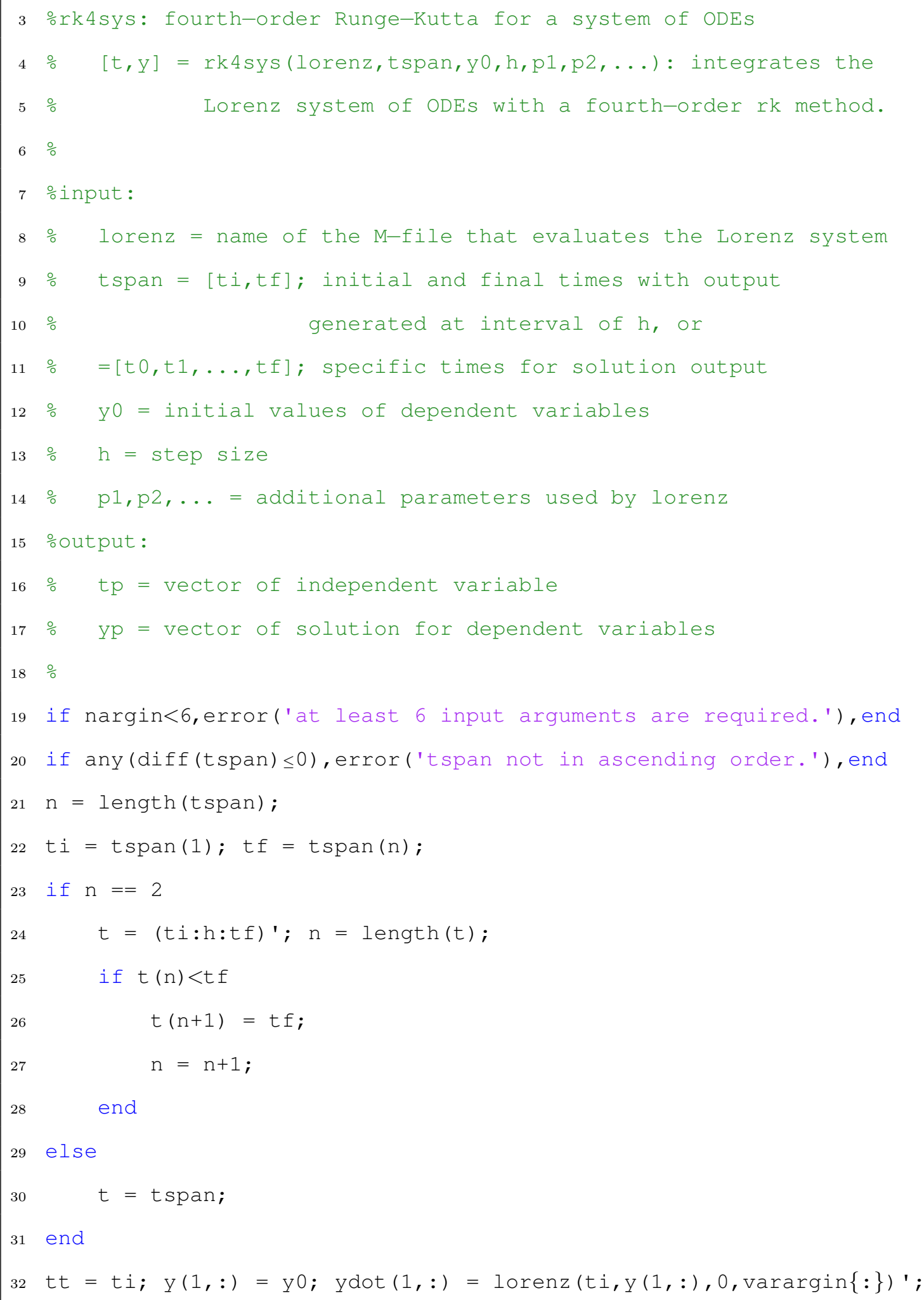




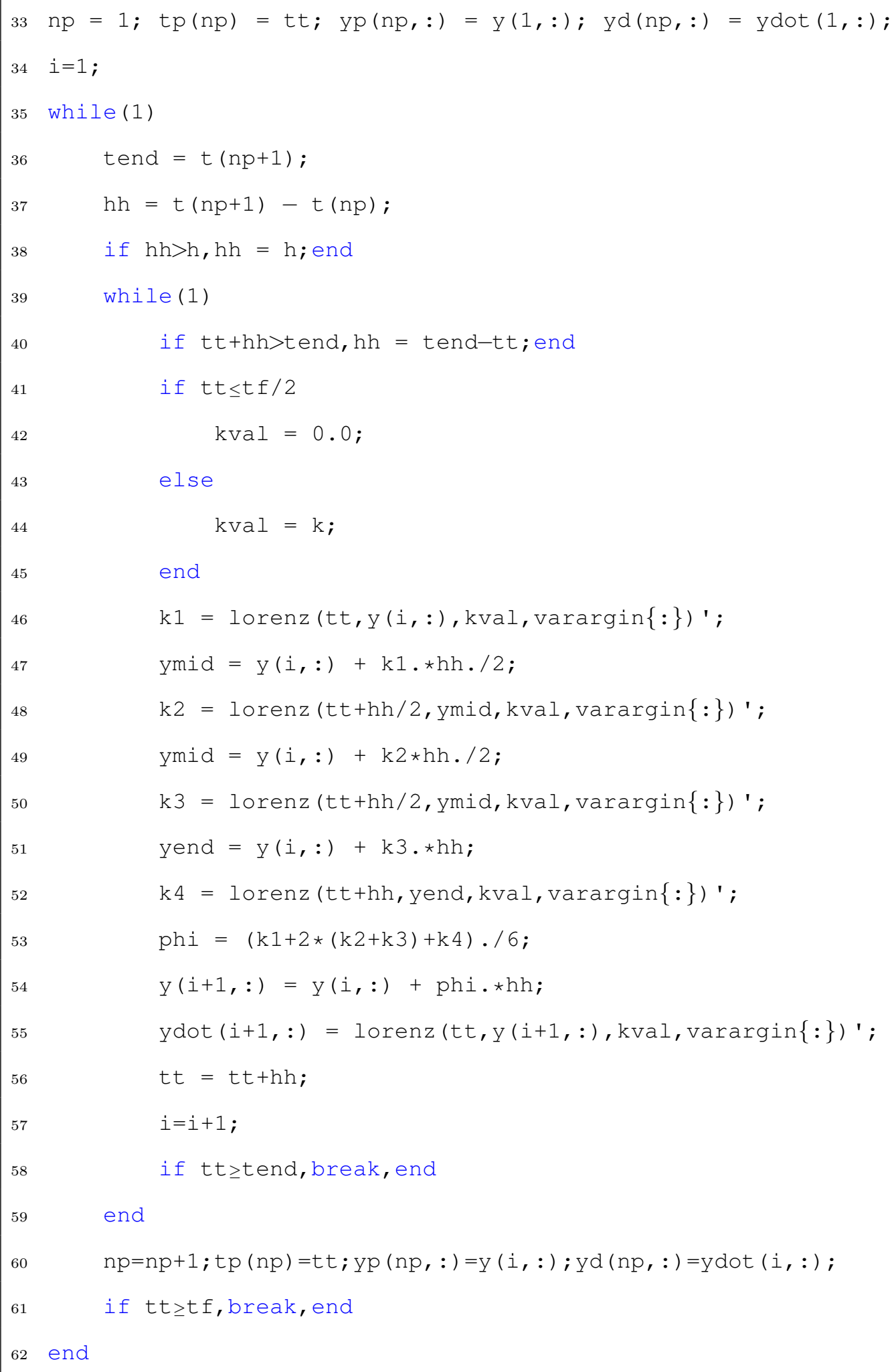


The Feigenbaum diagrams (such as Figure 2.4) were constructed using the code shown below.

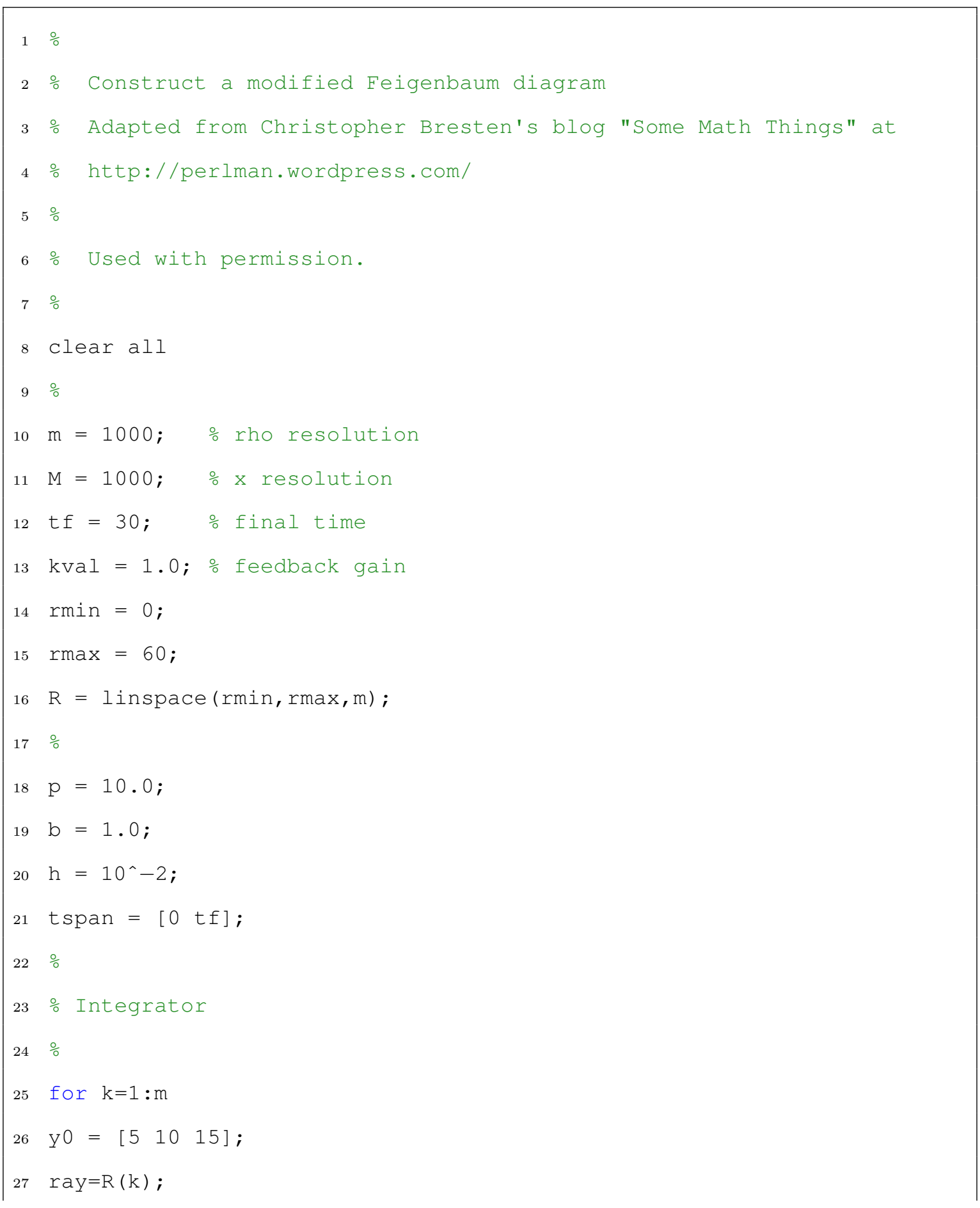




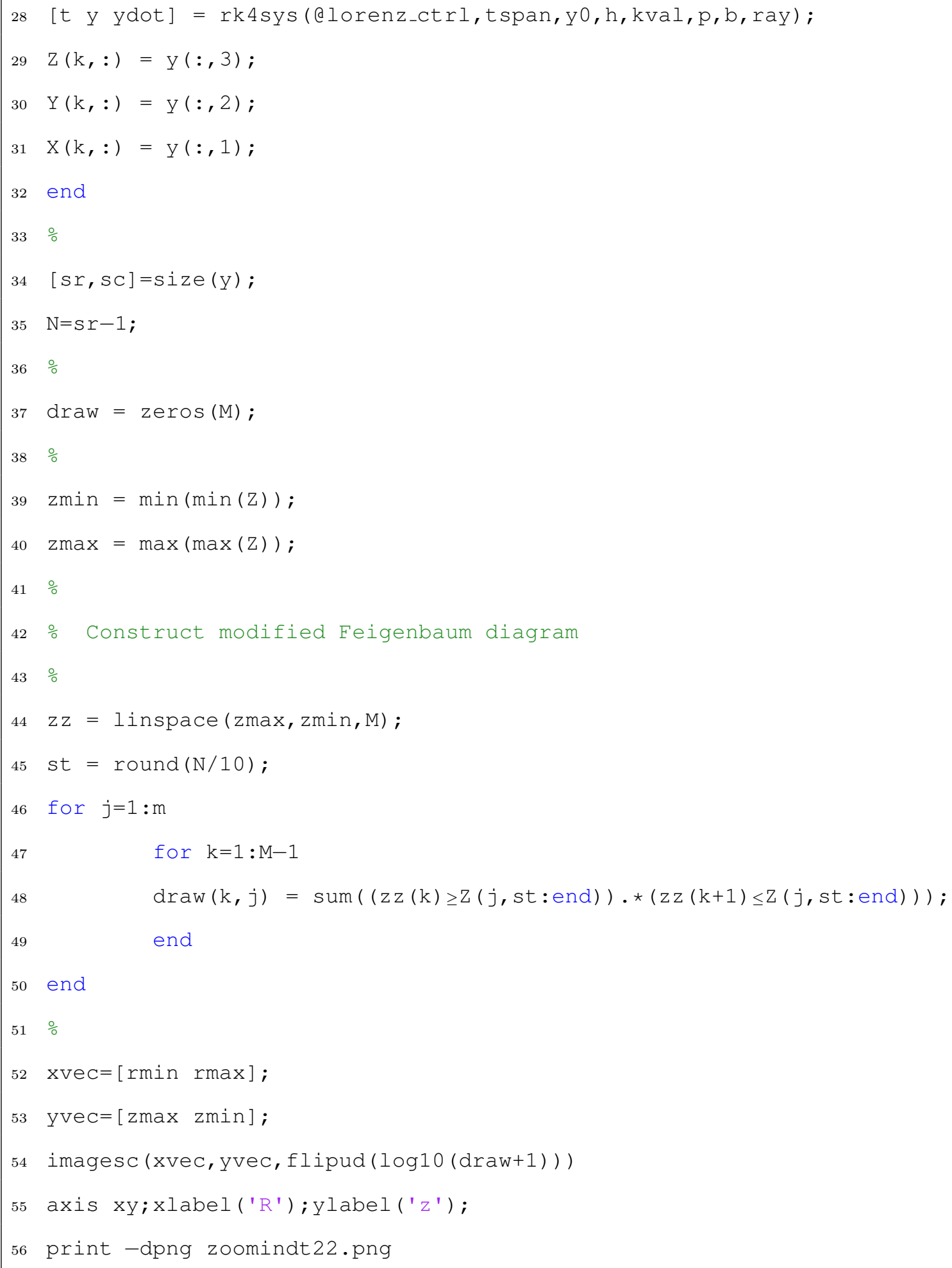


$4 \operatorname{phi}=\operatorname{acos}\left(-0.5 * \mathrm{k} / \operatorname{sqrt}\left(((\mathrm{R}-1) / 3)^{\wedge} 3\right)\right) ;$

$5 \quad x=2 * \operatorname{sqrt}((\mathrm{R}-1) / 3) * \cos (\mathrm{phi} / 3) ;$

$6 \quad c 2=20 * k^{\wedge} 2+(261-10 * R) * k * x+(242-110 * R) * x^{\wedge} 2+11 * k * x^{\wedge} 3+102 * x^{\wedge} 4$;

Finally, the Lozinskii norm in chapter 4 was evaluated using the code shown below.

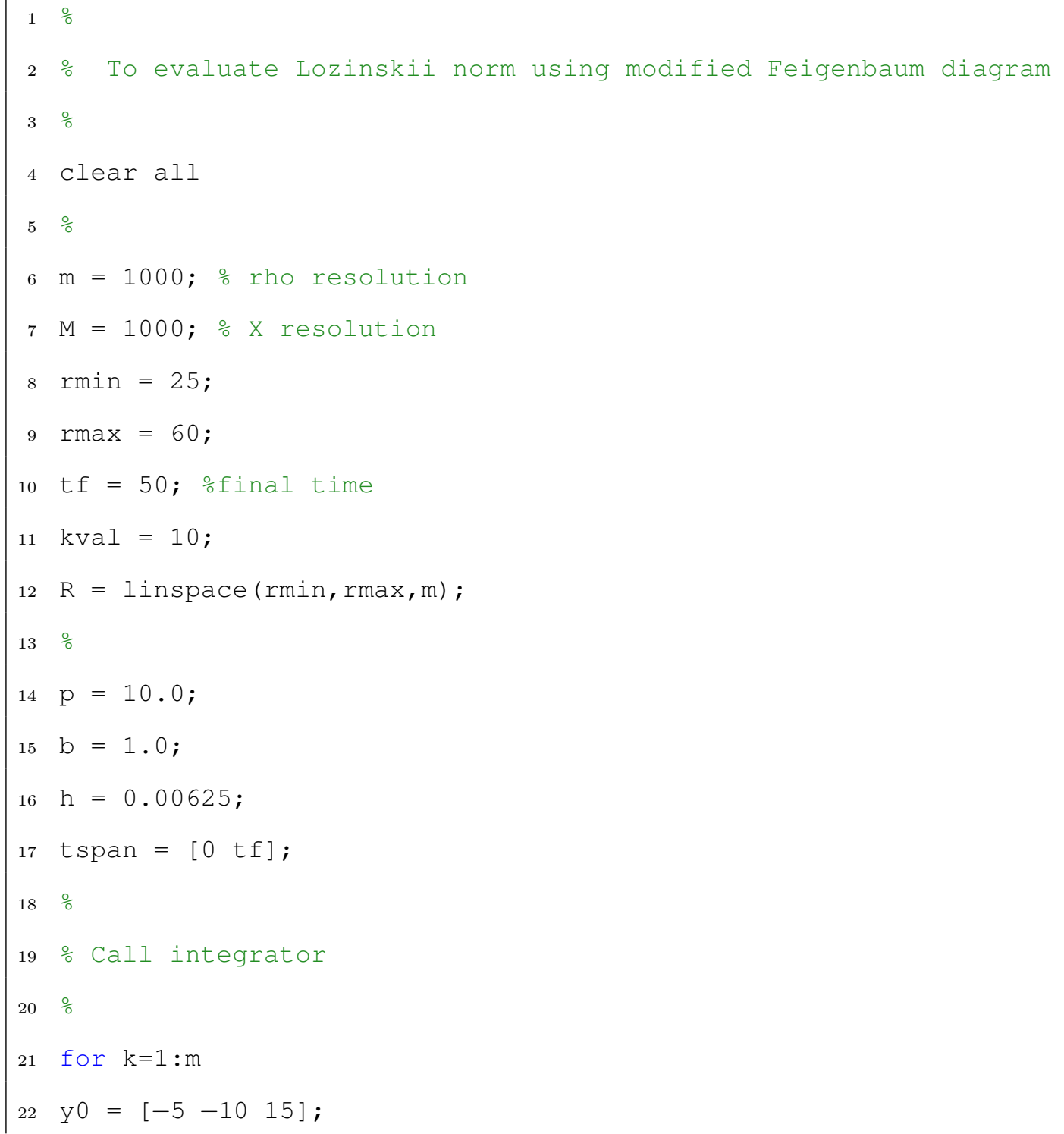




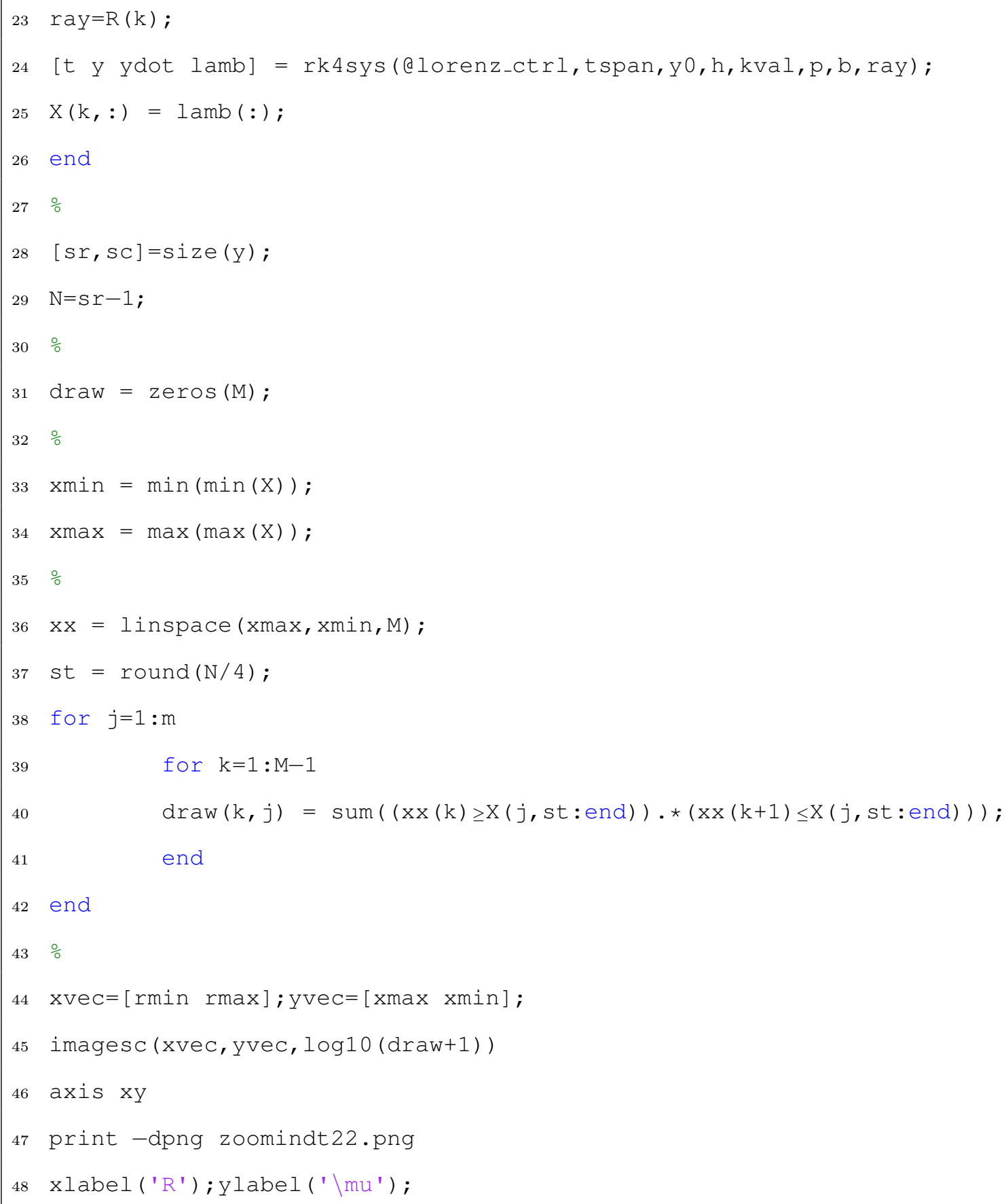

